\title{
Cyclophosphamide causes osteoporosis in C57BL/6 male mice: suppressive effects of cyclophosphamide on osteoblastogenesis and osteoclastogenesis
}

\author{
Dongfeng Zhao ${ }^{1,2,3, *}$, Chenglong Wang ${ }^{1,2,4, *}$, Yongjian Zhao ${ }^{1,2,3}$, Bing Shu ${ }^{1,2,3}$, Youji \\ Jia ${ }^{1,2}$, Shufen Liu ${ }^{1,2,3}$, Hongshen Wang ${ }^{1,2,3}$, Junli Chang ${ }^{1,2,3}$, Weiwei Dai ${ }^{1,4}$, Sheng \\ Lu $^{1,2}$, Qi Shi ${ }^{1,2,3}$, Yanping Yang ${ }^{1,2,3}$, Yan Zhang ${ }^{1,2,3}$ and Yongjun Wang ${ }^{1,2,3,5}$ \\ ${ }^{1}$ Longhua Hospital Affiliated to Shanghai University of Traditional Chinese Medicine, Shanghai, P.R. China \\ ${ }^{2}$ Spine Disease Research Institute, Shanghai University of Traditional Chinese Medicine, Shanghai, P.R China \\ ${ }^{3}$ Key Laboratory of Theory and Therapy of Muscles and Bones, Ministry of Education, Shanghai University of Traditional \\ Chinese Medicine, Shanghai, P.R. China \\ ${ }^{4}$ Central Laboratory of Research, Longhua Hospital, Shanghai, P.R. China \\ ${ }^{5}$ School of Rehabilitation Science, Shanghai University of Traditional Chinese Medicine, Shanghai, P.R. China \\ *These authors have contributed equally to this work \\ Correspondence to: Yongjun Wang, email: yjwang8888@126.com \\ Yan Zhang, email: medicineyan@aliyun.com \\ Yanping Yang, email: yanpingyangks@163.com
}

Keywords: cyclophosphamide; chemotherapy; osteoporosis; osteoblastogenesis; osteoclastogenesis

Received: May 09, $2017 \quad$ Accepted: August 23, $2017 \quad$ Published: September 18, 2017

Copyright: Zhao et al. This is an open-access article distributed under the terms of the Creative Commons Attribution License 3.0 (CC BY 3.0), which permits unrestricted use, distribution, and reproduction in any medium, provided the original author and source are credited.

\section{ABSTRACT}

The clinical evidence indicated that cyclophosphamide (CPD), one of the chemotherapy drugs, caused severe deteriorations in bones of cancer patients. However, the exact mechanisms by which CPD exerts effects on bone remodeling is not yet fully elucidated. Therefore, this study was performed to investigate the role and potential mechanism of CPD in osteoblastogenesis and osteoclastogenesis. Here it was found that CPD treatment $(100 \mathrm{mg} / \mathrm{kg} / \mathrm{day})$ for 7 days led to osteoporosis phenotype in male mice. CPD inhibited osteoblastogenesis as shown by decreasing the number and differentiation of bone mesenchymal stem cells (MSCs) and reducing the formation and activity of osteoblasts. Moreover, CPD suppressed the osteoclastogenesis mediated by receptor activator for nuclear factor-K B ligand (RANKL) as shown by reducing the maturation and activity of osteoclasts. At the molecular level, CPD exerted inhibitory effect on the expression of components (Cyclin D1, $\beta$-catenin, Wnt 1, Wnt10b) of Wnt/ $\beta$-catenin signaling pathway in MSCs and osteoblasts-specific factors (alkaline phosphatase, Runx2, and osteocalcin). CPD also down-regulated the expression of the components (tumor necrosis factor receptorassociated factor 6 , nuclear factor of activated T-cells cytoplasm 1, c-Fos and NF-kB) of RANKL signaling pathway and the factors (matrix metalloproteinase 9, cathepsin $K$, tartrate-resistant acid phosphates and carbonic anhydrase II) for osteoclastic activity. Taken together, this study demonstrated that the short-term treatment of CPD induced osteoporosis in mice and the underlying mechanism might be attributed to its marked suppression on osteoblastogenesis and osteoclastogenesis, especially the effect of CPD on bone formation might play a dominant role in its detrimental effects on bone remodeling. 


\section{INTRODUCTION}

Cancers cause severe mortality and lead to significant societal burdens globally. The chemotherapy represents one of the most effective strategies in treating types of cancers [1]. Cyclophosphamide (CPD), one class of drugs from alkylating agent family, increases the survival rate of cancer patients $[2,3]$ by inhibiting the proliferation of tumor cells via intercalating with DNA $[4,5]$. However, CPD also causes severe side effects on the skeleton, leading to increased risks of osteoporosis $[6$, 7]. In addition, it was reported that the treatments with either CPD or CPD regiments induced the early onset of menopause in women, and consequently led to the occurrence of osteoporotic phenotype at both at trabecular bone and cortical bone $[8,9]$.

The available evidence demonstrated that the osteoporotic phenotype in cancer patients treated with CPD is caused, at least partially, by hypogonadism [6, $10]$, because CPD causes damages on ovaries in women and decreases levels of androgens in men [9-12]. The failure of ovaries leads to the decrease of estradiol (E2) production similar to that seen in postmenopausal osteoporosis [13]. Furthermore, CPD exerted inhibitory effect on bone formation by arresting the differentiations of preosteoblasts [14] and increased the level of folliclestimulating hormone (FSH) and luteinizing hormone (LH) [15-17], which are inhibitors on bone formation. Additionally, several studies have reported that the CPD regiments enhanced the expression of bone resorption markers in long bones of female rats [18-20], promoted the differentiation of mesenchymal stem cells (MSC) to adipocytes $[18,21]$, and induced bone resorption by altering the ratio of receptor activator for nuclear factor- $\kappa$ $\mathrm{B}$ ligand (RANKL)/osteoporotegerin (OPG) in serum of early-stage breast cancer patients [12]. Thus, CPD-induced osteoporosis may be attributed to its damages on sex gland and bone formation and resorption. However, the underlying mechanism for the effect of CPD on osteoblast and osteoclast is yet to be determined.

$\mathrm{Wnt} / \beta$-catenin plays critical roles in regulating osteoblastogenesis [22] and bone formation [23]. Wnt family members, as such as Wnt1 and Wnt10b, are secreted cysteine-rich glycoproteins in osteoblast and interact with $\beta$-catenin to activate Wnt signaling [24, $25]$. The activation of $\mathrm{Wnt} / \beta$-catenin promotes the differentiation of osteoblast from mesenchymal stem cells (MSCs) by stimulating the expression of Cyclin D1 and

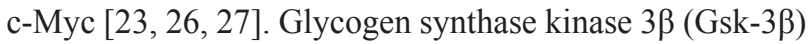
plays crucial roles in regulating the activity of canonical Wnt signaling pathway [28]. Normal expression of Gsk$3 \beta$ inhibits the combination of Wnt ligand to $\beta$-catenin through activating the phosphorylation of $\beta$-catenin [29]. In contrast, the stimulated expression of Gsk-3 $\beta$ increases the ubiquitination and proteosomal degradations of $\beta$-catenin thus to reduce the translocation of $\beta$-catenin into the nucleus and secondly inhibits osteoblast differentiation from MSCs [30, 31]. Furthermore, Wnt/ $\beta$-catenin pathway works in collaborating with several osteoblast-related regulators, including ALP, Runx2, and osteocalcin [32, 33], which are necessary for osteoblastogenesis.

Osteoclasts differentiate from bone marrow macrophages (BMMs) upon stimulation by two essential cytokines, macrophage colony stimulating factor (M-CSF) and receptor activator for nuclear factor- $\kappa$ B ligand (RANKL) [34]. RANKL binds to its receptor RANK, a member of the TNF receptor super family, to recruit TNF receptor-activated factors family members (TRAF-1, 2, $3,4,6)[35,36]$. The recruitments of TRAFs activates numerous signaling pathways (IKB $\alpha / \mathrm{NF}-\mathrm{\kappa B}, \mathrm{ERK}, \mathrm{JNK}$, p38) which are necessary for osteoclastogenesis [37, 38]. Meanwhile, RANKL also stimulates the expression of nuclear factor of activated T-cells-cytoplasm 1(NFATc1) [38] and c-Fos [39] as well as osteoclast genes, such as those encoding matrix metalloproteinase 9 (MMP9), cathepsin $\mathrm{K}$ (Ctsk), tartrate-resistant acid phosphates (TRAP) and carbonic anhydrase II (Car2) [40]. The biological influences of CPD on the osteoclastogenesis are not clearly known, even though some studies indicated that CPD suppressed bone resorption by arresting the proliferation of osteoclasts [6] and the combination chemotherapy with CPD, epirubicin and 5-fluorouracil stimulated the RANKL-mediated osteoclatsogenesis [18]. Therefore, it is necessary to investigate the exact roles of CPD in regulating RANKL-mediated osteoclastogenesis.

The earlier studies demonstrated that the anti-tumor effects of CPD were caused by its regulation on Wnt/ $\beta$ catenin pathway $[41,42]$. One recent study found that CPD exerted anti-tumor effects in sarcoma S180 tumorbearing mice by down-regulating the expression Wnt/ $\beta$ catenin and Cyclin D1 [41]. Additionally, CPD exerted inhibitory effects on angiogenesis in Lewis lung cancer mice through negatively modulating the $\mathrm{Wnt} / \beta$-catenin signaling pathway [42]. To our knowledge, the direct effects of CPD on Wnt/ $\beta$-catenin during the process of osteoblastogenesis are not yet investigated $[6,43]$.

This study sought to investigate the effects of $\mathrm{CPD}$ on bones in vivo using mouse models. Moreover, in vitro assays were performed to investigate the effects of CPD on the differentiation and activity of osteoblasts and osteoclasts. Finally, in vitro mechanistic studies were carried out to elucidated the mechanism by which CPD exerts its effects on osteoblast and osteoclast differentiation.

\section{RESULTS}

\section{Identification of 4-OH-CPD in conditioned medium (CM)-CPD working solution}

The structures of CPD and 4-OH-CPD are shown in Figure 1A. 4-OH-CPD is the active intermediate metabolite. 
The concentration of 4-OH-CPD is usually used as one critical marker to monitor the efficacy of CPD-based chemotherapy and therefore the liquid chromatography mass spectrometry (LC-MS/MS) analysis were carried out. Briefly, the concentration of 4-OH-CPD in CPD-H (4000 $\mu \mathrm{M})$ was detected in multiple reactions monitoring (MRM) mode. The peak area (Figure 1B) represented the quality or concentration of 4-OH-CPD. The calculated concentration of 4-OH-CPD in CPD-H $(4000 \mu \mathrm{M})$ was about $175.3 \mathrm{ng} /$ $\mathrm{mL}(0.6 \mu \mathrm{M})$ based on the standard curve (Figure $1 \mathrm{C})$. This study identified and quantified the active 4-OH-CPD in conditioned medium (CM)-CPD working solution, which confirmed and guaranteed the biological influences of CPD in the following in vitro studies.

\section{CPD treatment induced the phenotype of osteoporosis}

Numerous clinical observations have demonstrated that the chemotherapy treatment with CPD caused osteoporosis phenotypes. This study was conducted to firstly confirm whether CPD exerts similar effects in mice. Male C57BL/6 mice were treated with CPD (100 $\mathrm{mg} / \mathrm{kg} / \mathrm{d}$, i.p.) or saline as control for 7 consecutive days. The mice were sacrificed at 3, 7 and $12 \mathrm{~d}$ after CPD injection. To exclude the systematic toxicity, the changes of body weight were measured during experimental period. The body weights of CPD-treated mice were significantly decreased $(P<0.05)$ at $3 \mathrm{~d}$ and $7 \mathrm{~d}$ during the CPD treatment period. While, the body weight at $12 \mathrm{~d}$ in CPD group restored to the same level as that in the saline control group (Supplementary Figure 1). Moreover, the animal experiments with three independent times were performed (12 mice/group) and no mice were dead during the experiment. Therefore, CPD did not induce systemic toxicity in this study.

Lumbar spine (LV1 3) and proximal end of tibia measurement were scanned by micro-CT to obtain skeletal phenotype following the instruction of the ASBMR guideline in 2010 [44]. The result indicates that CPD-

\section{A}<smiles>O=P1(N(CCCl)CCCl)NCCCO1</smiles>

CPD<smiles>O=P1(N(CCCl)CCCl)NC(O)CCO1</smiles>

4-OH-CPD
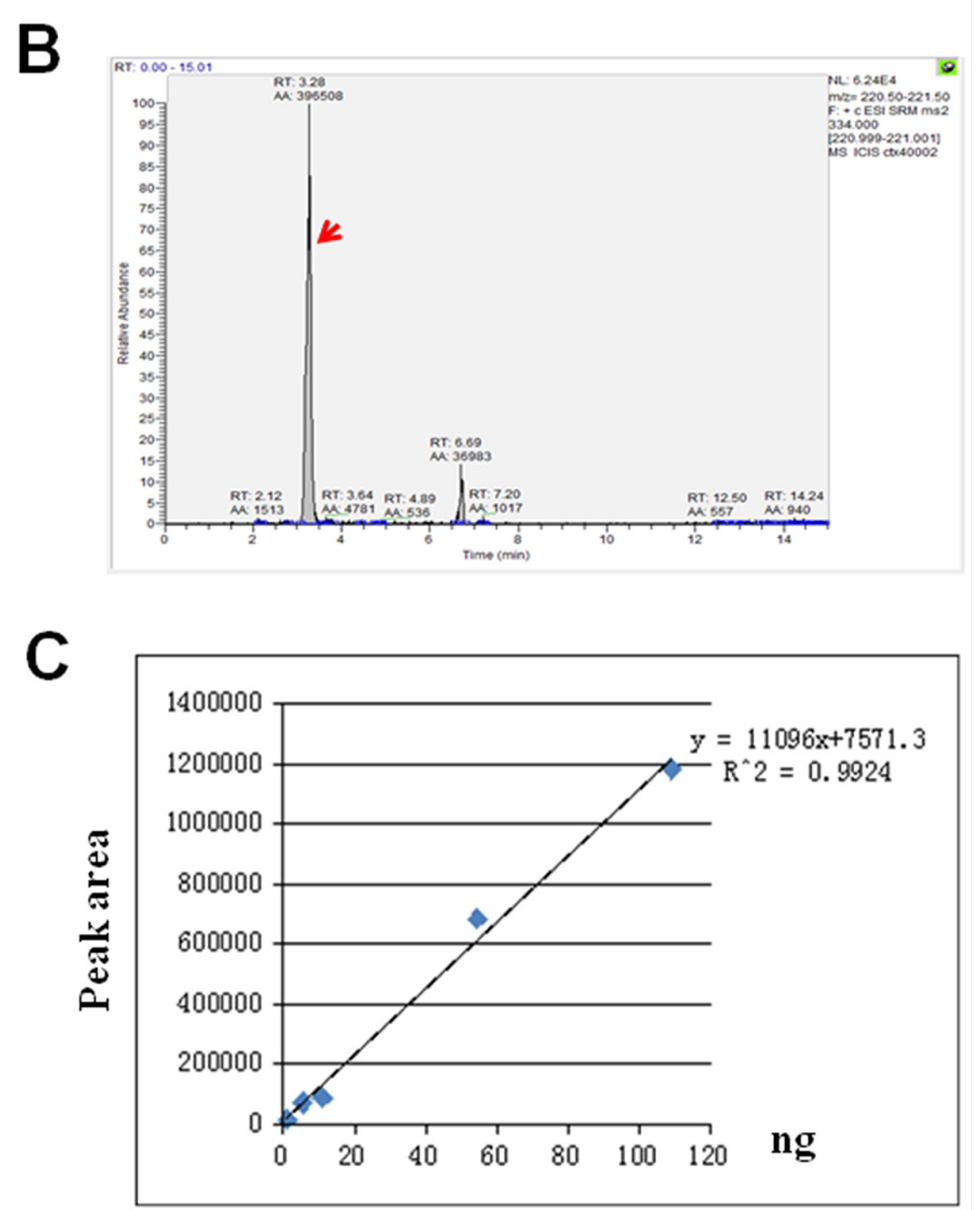

Figure 1: The identification of conditioned medium (CM)-CPD working solution. (A) Chemical structure of cyclophosphamide (CPD) and its medial metabolic product (4-OH-CPD) that can be detected in the conditioned medium working solution. (B) The LC/MS/ MS chromatograms of 4-OH-CPD (as indicated by the arrow) in CPD-H $(4000 \mu \mathrm{M})$ in multiple reaction monitoring (MRM) mode. (C) Standard curve were made with 10 times serial dilution of 4-Hydroperoxy CPD. 
intervention caused significant osteoporosis phenotypes both at lumbar spine (LV1 3) (Figure 2A) and proximal end of tibia (Figure 2D). Compared to saline control group, the application of the CPD caused significant decreases in bone mineral density (BMD), connectivity density (Conn. $\mathrm{D})$, trabecular bone number $(\mathrm{Tb} . \mathrm{N})$ and trabecular bone thickness (Tb. Th) of LV1 3 (Figure $2 \mathrm{~B}$ ) at day $3(P<0.05)$, $7(P<0.05)$ and $12(P<0.01)$. In addition, CPD treatment resulted in the decrease of $\mathrm{BMD}$, Conn. $\mathrm{D}$, trabecular bone volume over total volume (BV/TV), Tb. N and Tb. Th as well as the increase of trabecular bone separation (Tb. Sp). Moreover, these alterations of trabecular bone parameters were most significant $(P<0.01$, Figure $2 \mathrm{E})$ at day 12 . The changes of these quantitative parameters of trabecular bone at LV1 3 and tibia were in accordance with the histological images by $\mathrm{H} \& \mathrm{E}$ staining (Figure $2 \mathrm{C}$ and $2 \mathrm{~F}$ ) which clearly showed the loss of trabecular bone number and network. Thus, CPD treatment induced the severe osteoporotic phenotype at trabecular bone of male mice.

\section{CPD down-regulated the expression of osteoblast differentiation-related factors}

To explore the effects of CPD on the expression of a series of osteoblast differential-related biomarkers, we first detected the protein expression of Runx2 (Figure 3A) and $\beta$-catenin (Figure 3B) in tibia of CPD-treated mice at day 12. Meanwhile, the protein expression of $\beta$-catenin, Cyclin D1, and Runx2 were assessed with LV4 5 from CPD-treated or saline control mice (Figure 3C and 3D). Next, the mRNA expression of Runx2, $\beta$-catenin, Osteocalcin, ALP, Wnt 1, Wnt4, Wnt10b and Cyclin D1 in lumbar vertebra (Figure 3E). CPD treatment largely decreased the positive signals of Runx 2 and $\beta$-catenin as well as markedly reduced the mRNA expression of Runx2, ALP, $\beta$-catenin, Osteocalcin, Wnt 1, Wnt4, Wnt10b and Cyclin D1 of LV4 5 $(P<0.01)$.

\section{CPD treatment decreased the number of bone mesenchymal stem cells}

Bone primary mesenchymal stem cells were isolated and flushed with $\alpha$-MEM from the bilateral femur and tibia of naïve, saline control or CPD-treated male C57BL/6 mice. Mature osteoblasts were derived from bone marrow mesenchymal stem cells, the number of which was determined and compared in CPD-treated or saline control mice. We next fully examined the effects of CPD on the number of MSCs by assessing the expression of CD29 ${ }^{+}$, $\mathrm{CD}_{4}{ }^{+}$, and CD45- which are critical markers for MSCs [45]. The percentage of CD45- is $14.73 \%$ in saline control mice but is $0.6 \%$ in CPD-treated mice. In addition, the percentage of $\mathrm{CD} 29^{+} \mathrm{CD} 44^{+} \mathrm{CD} 45^{-}$is $9.17 \%$ in saline control and is $0.2 \%$ in CPD-treated group (Figure 4A). Furthermore, statistical analysis indicates that the number of MSCs per CPD-treated mice is much lower than saline control (Figure 4B).

\section{CPD inhibited cell viability and osteoblastic differentiation of MSC}

Osteoblasts differentiate from bone MSCs. Normal viability of MSCs and osteoblastic differentiation are requirements for $\mathrm{BMD}$ and bone function. We further investigated the roles of CPD in cell viability of MSCs and osteoblastogenesis. MSCs from male C57/BL mice (Figure 4C) and C3H10T1/2 cell lineage (Figure 4D) were treated with different concentrations of CPD working solutions (CPD-Con, CPD-D and CPD-H) to measure the cell viability. CPD dose-dependently decreased the cell viability of primary bone MSCs and C3H10T1/2 cells and the most significant suppressive effect was observed at time point of 72 hours.

The differentiation of MSCs to osteoblasts plays a key role in regulating bone formation. Bone MSCs were treated with CPD-Con, CPD-B, CPD-D and CPD-H, and then stained for ALP. The results showed that CPD dosedependently inhibited the amount of ALP-positive staining in bone MSCs (Figure 4E). Meanwhile, both Alizarin Red (Figure 4F, above panel) and ALP staining (Figure $4 \mathrm{~F}$, bellow panel) demonstrated that there were decreased osteoblastogenesis of bone MSCs, collected at day 3 and day 7 , in CPD-treated mice.

\section{CPD suppressed osteoblastogenesis by down- regulating Wnt/ $\beta$-catenin pathway}

Wnt/ $\beta$-catenin pathway plays important roles in stimulating osteoblastogenesis. The activity of $\mathrm{Wnt} / \beta$ catenin signaling was investigated (Figure 5). The realtime PCR result showed that CPD significantly suppressed mRNA levels of Wnt1, Wnt4, and $\beta$-catenin in LV4 5 of CPD-treated mice as compared to those of saline control group $(P<0.01$, Figure 5A). Furthermore, primary bone MSCs were isolated from saline- or CPD-treated (100 mg/ $\mathrm{kg}$ ) mice, and the western blot showed that the protein expression of active $\beta$-catenin, Runx2, and Cyclin D1 was significantly decreased, and DDk1 protein expression was markedly increased in CPD-treated group (Figure 5B and $5 \mathrm{C}$ ). Next, we further investigated the effects of CPD exerts on a series of markers during osteoblastogenesis at different time points $(0,3,6,12,24,48 \mathrm{~h})$ in primary bone MSCs, such as the expressions of active $\beta$-catenin, Runx2, and Cyclin D1 and c-Myc. Our finding indicates that CPD significantly inhibits the expression of $\beta$-catenin, Runx 2 , Cyclin D1 and c-Myc (Figure 5D and 5E).

Furthermore, we investigated whether CPD exerts inhibitory effects on the expression of total and nuclear $\beta$-catenin. C3H10T1/2, one common MSCs cell line, were treated with CPD and the total and nuclear proteins were assessed by western blot analysis. Our result showed that CPD both exerts inhibitory effects on the expression of both total and nuclear $\beta$-catenin (Figure 5H and 5I). Next, $\mathrm{C} 3 \mathrm{H} 10 \mathrm{~T} 1 / 2$ was used to assess the expression of $\beta$-catenin 

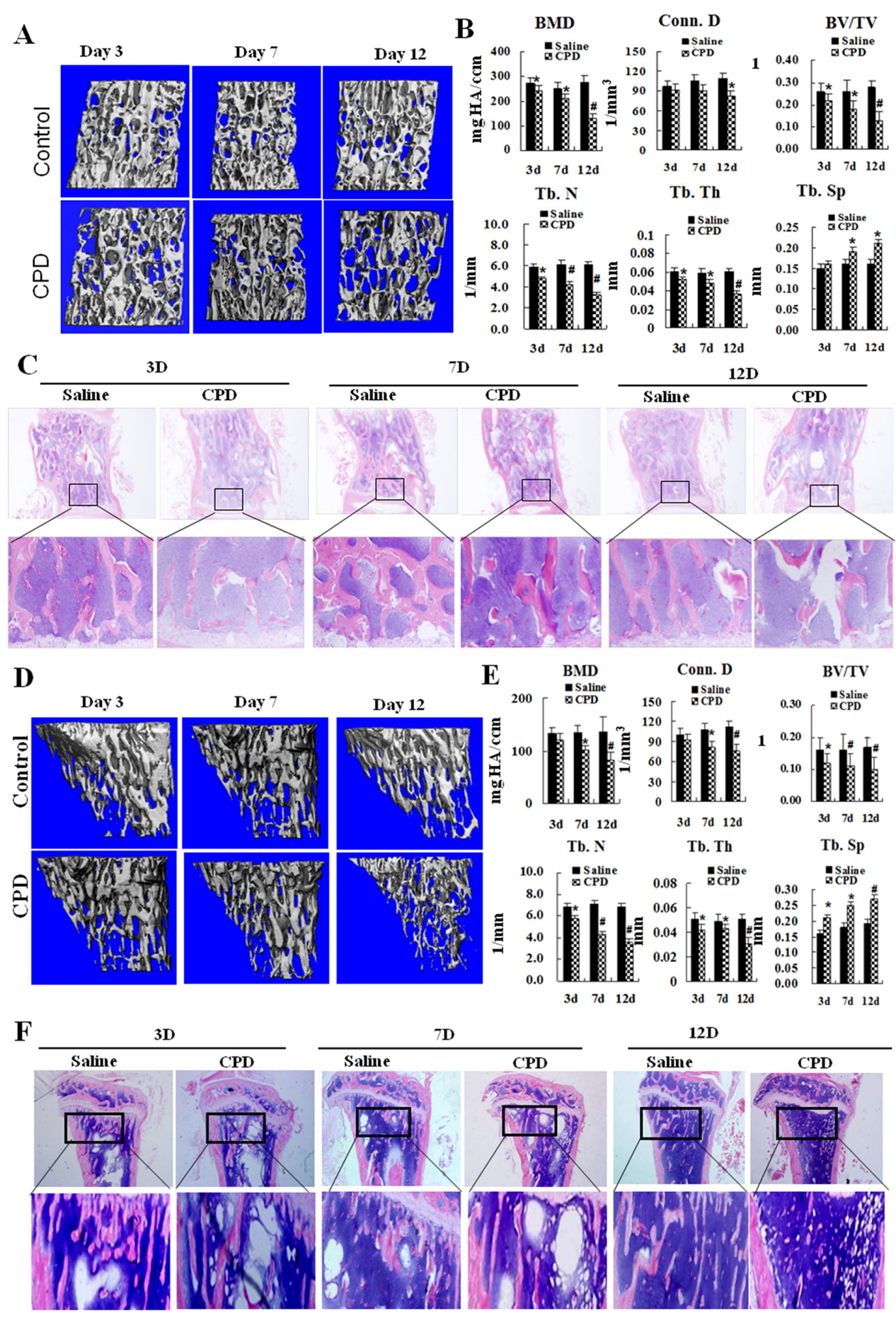

Figure 2: The treatment with cyclophosphamide caused severe bone loss in mice. (A) Male C57BL/6 mice were administered with saline or cyclophosphamide (CPD, $100 \mathrm{mg} / \mathrm{kg} /$ days (d)) for 7 consecutive days by intraperitoneal injections. The mice were maintained for additional 3,7and 12 days before harvesting. Micro-CT analysis was performed on lumbar vertebrate (LV1 3) and one representative imagine from each group was shown. (B) The bone parameters were shown as the following: Bone mineral density (BMD); Connectivity density (Conn. D); Bone volume over total volume (BV/TV); Trabecular bone number (Tb.N); Trabecular bone separation (Tb. Sp); Trabecular bone thickness (Tb. Th). (C) H\&E stains were performed on LV1 3. (D) One representative image on the proximal metaphysis of tibia was shown. (E) The bone parameters were shown as the following: Bone mineral density (BMD); Connectivitydensity (Conn. D); Bone volume over total volume (BV/TV); Trabecular bone number ( $\mathrm{Tb}$. N); Trabecular bone separation ( $\mathrm{Tb}$. Sp); Trabecular bone thickness (Tb. Th). (F) H\&E stains were performed on tibias. Values were expressed as means \pm SEM. ${ }^{*} P<0.05,{ }^{"} P<0.01$, vs. Saline control group at the same time point. 
activated signaling in osteoblastogenesis. The result shows that CPD significantly suppressed the expression of active $\beta$-catenin, Runx2, c-Myc, and Cyclin D1; however, CPD stimulates the expression of phospho-Gsk-3 $\beta$ (Figure $5 \mathrm{~F}$ and $5 \mathrm{G}$ ), which are critical markers for osteoblastogenesis. In addition, we also determined the expression of Runx2 and Cyclin D1 with RNA by C3H10T1/2. To our expectation, CPD inhibited the expressions of Runx 2 and Cyclin D1 in a time-dependent manner (Figure 5J).

\section{CPD decreased the number of TRAP-positive mature osteoclasts in vitro osteoclast assays}

Bone remodeling is modulated by the tight coupling between osteoblast mediated bone formation and osteoclasts mediated bone resorption. Since CPD exerted inhibitory effects on osteoblastogenesis, it was interesting to investigate the effects of CPD on osteoclastogenesis and bone resorption.

Male C57BL/6 mice were treated with CPD (100 $\mathrm{mg} / \mathrm{kg} / \mathrm{d}$, i.p.) or saline as control for 7 consecutive days. Subsequently, the mice were sacrificed at 3, 7 and $12 \mathrm{~d}$ after CPD treatment. TRAP activity in distal femoral metaphysis (Figure 6A) was less observed in CPD-treated mice at 3, 7 and $12 \mathrm{~d}$ as compared to those of saline control, and the CPD treatment significantly reduced the number of osteoclasts-covered surface (Figure 6B) and osteoclasts number (Figure 6C) in femur. These findings indicated that the osteoclastic resorptive activity was greatly depressed in CPD-treated mice.

To further confirm the inhibitory effect of CPD on RANKL-mediated osteoclastogenesis, we next carried out ex vivo assays with Bone marrow macrophages (BMMs, namely
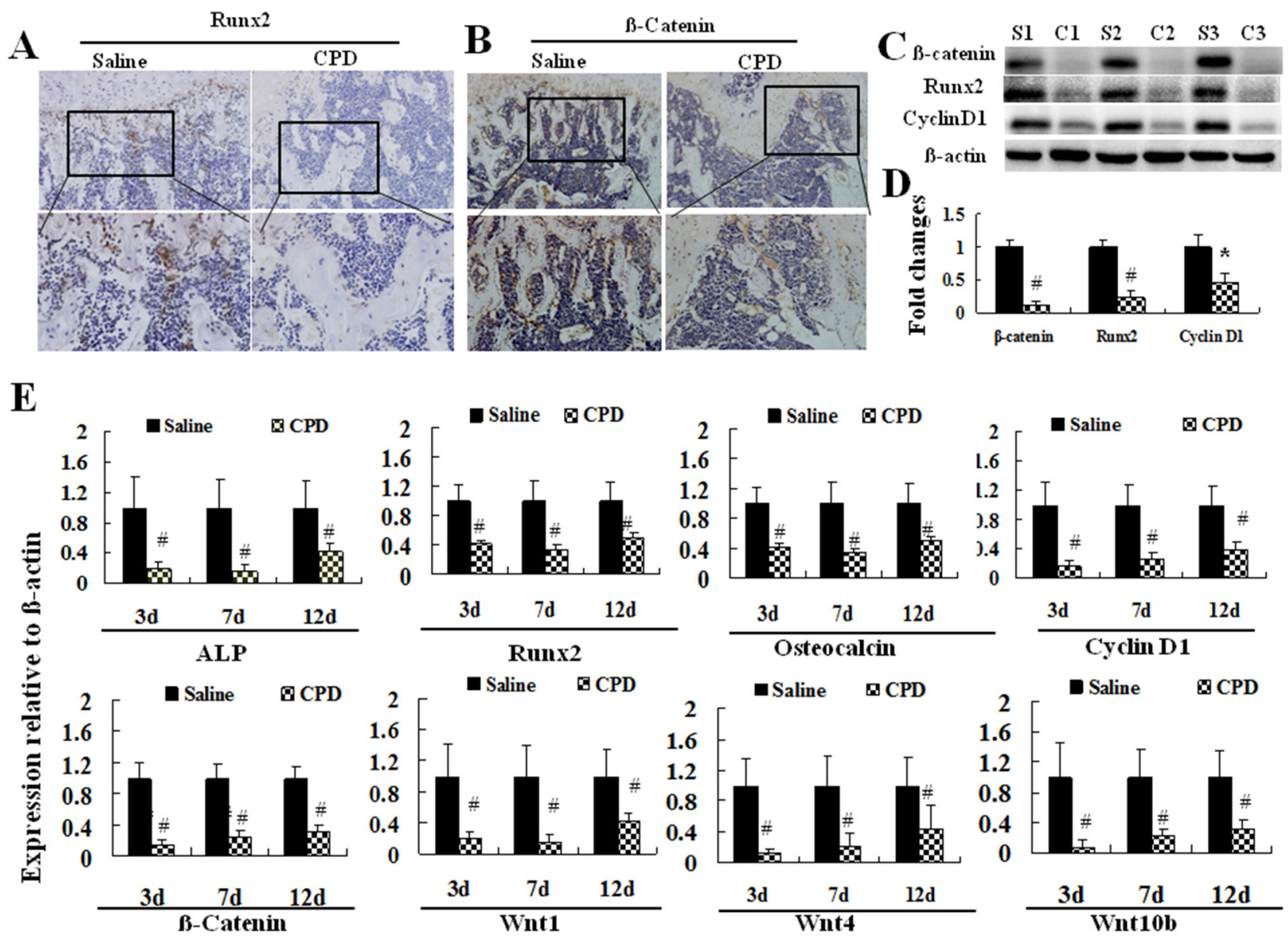

Figure 3: Cyclophosphamide exerted inhibitory effects on the expression of osteoblast differentiation-related factors. The male C57BL/6 mice were administered with saline or cyclophosphamide (CPD, 100mg/kg/d) for 7 consecutive days by intraperitoneal injection. The tibia was harvested, decalcified and fixed to make paraffin sections after the mice were maintained for additional 12 days. The protein expression of Runx2 (A) and $\beta$-catenin (B) were assessed by immunohistological (IHC) staining. (C, D) LV4 5 from CPDtreated or saline control mice, randomly at least 3 mice each group, were harvested from at 12 days after intervention of CPD. The protein expressions of $\beta$-catenin, Runx2, and Cyclin D1 were assessed with western blot (C) and quantified. (D), ${ }^{*} P<0.05,{ }^{\#} P<0.01$, vs. saline. S:saline, C:CPD. (E) Real-time PCR was performed to assess the mRNA expression of Runx2, $\beta$-catenin, Osteocalcin, alkaline phosphatase (ALP), Wnt1 and Cyclin D1 with mRNA from LV4 5. Values were expressed as means \pm SEM. ${ }^{\#} P<0.01$, vs. saline control group. At least 3 mice in each group were performed for IHC assays, and Real-time PCR assays. 
osteoclast precursors) from CPD-treated mice or saline control. M-CSF and RANKL are two essential and sufficient factors for osteoclastogenesis [34]. Primary BMMs were treated with M-CSF (44 ng/ml) and RANKL (100 ng/ml) for 5 days before performing TRAP staining (Figure 6D). BMMs from saline control differentiated to TRAP-positive osteoclasts, but few from CPD-treated mice $(P<0.01$, Figure $6 \mathrm{E})$, indicating that CPD-treatment impaired osteoclast differentiation.

\section{CPD dose-dependently inhibited osteoclastogenesis}

Next, osteoclastogenesis assays were performed with BMMs from wide-type $\mathrm{C} 57 \mathrm{BL} / 6$ mice. BMMs that treated with M-CSF and RANKL as positive control. Meanwhile, BMMs were treated with M-CSF and RANKL plus CPD-
Con, or CPD-A, or CPD-B, or CPD-C or CPD-D for 5 days before performing TRAP staining. As the results shown in Figure 6F, both positive control and CPD-Con formed numerous osteoclast. In contrast, there were fewer osteoclasts existed in CPD-treated group and no osteoclasts were observed in CPD-D $(2000 \mu \mathrm{M}, P<0.001)$. These results indicated that $\mathrm{CPD}$ dose-dependently inhibited RANKL-mediated osteoclastogenesis.

To select the proper concentration of CPD, we further assessed the viability of BMMs in the presence of CPD working solutions without RANKL to exclude the cytotoxicy of CPD. BMMs were treated in the presence of $\mathrm{M}-\mathrm{CSF}$ alone plus each CPD working solutions (Control, CPD-Con, CPD-A to CPD-H) for 5 days before performing cell viability assays with cell counting kit (CCK-8). However, there were no significance of the percentages of
A
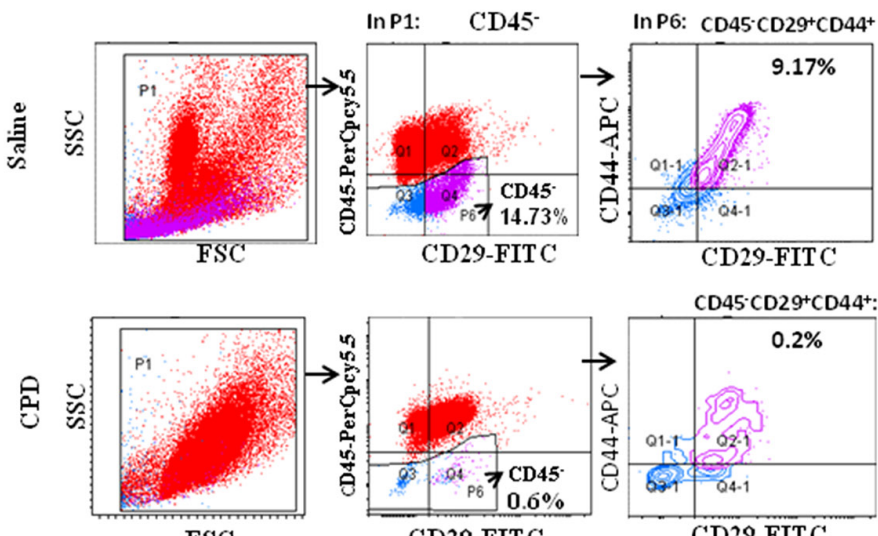

FSC

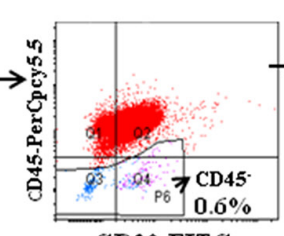

CD29-FITC
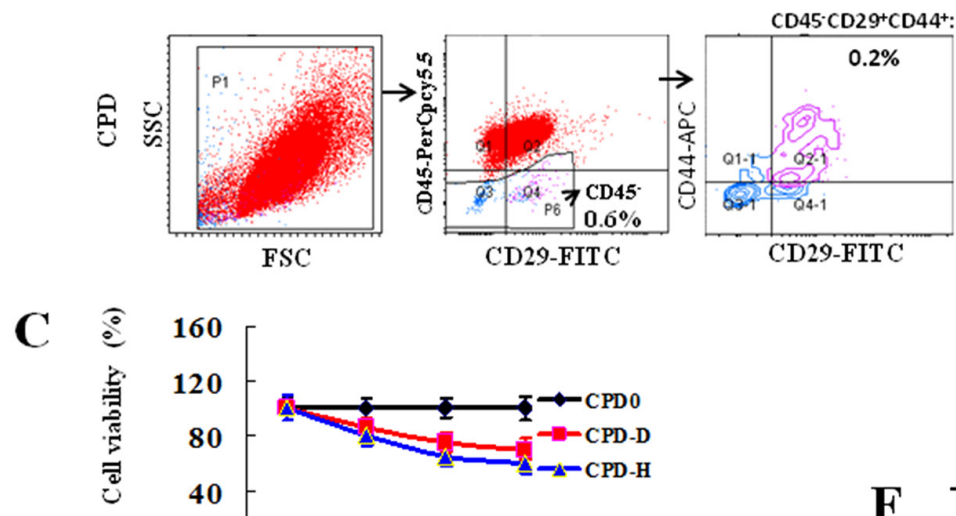

$\mathbf{E}$

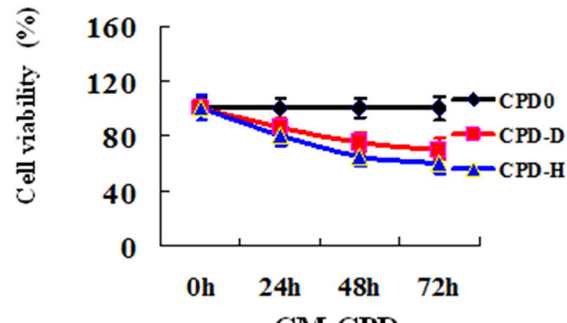

CM-CPD

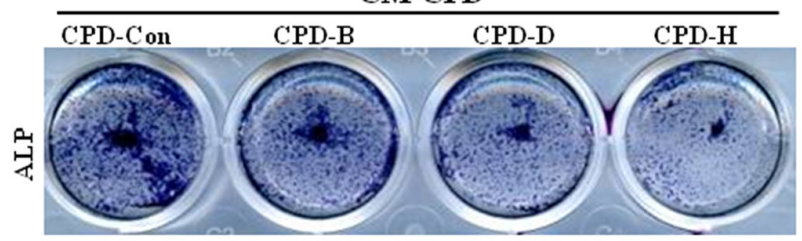

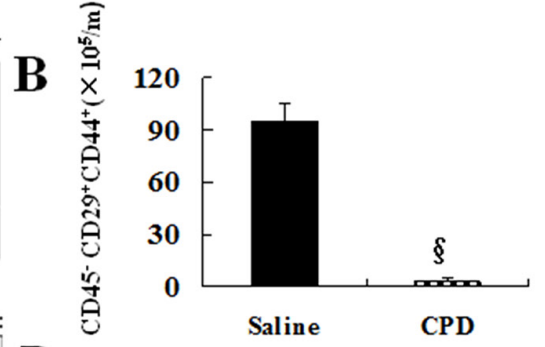

$\mathbf{D}$
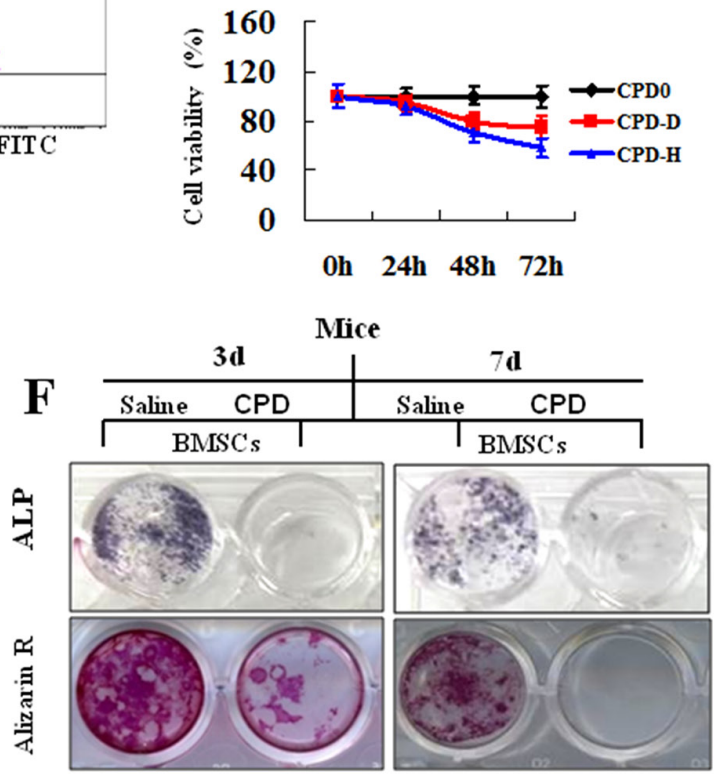

Figure 4: Cyclophosphamide decreased the number of mesenchymal stem cells, cell viability and capability of osteoblastogenesis. (A) Bone mesenchymal stem cells (MSCs) from tibia/femur in saline or CPD-treated male mice were prepared. The expressions of positive $\mathrm{CD} 29^{+}, \mathrm{CD} 44^{+}$, and $\mathrm{CD}^{-} 5^{-}$were tested by the fluorescence activating cell sorter (FACS). (B) The number of MSCs from per mice $(\mathrm{m})$. Primary bone MSCs (C) or C3H10T1/2 lineages (D) were treated with CPD-Con, CPD-D or CPD-H working solutions for 0, 24, 48 and 72 hours before measuring cell viability. (E) Primary bone MSCs, isolated from the bilateral femur/tibia of male C57BL/6 mice, was cultured with CPD-Con, CPD-B, CPD-D and CPD-H for 2 days. All the assays were performed alkaline phosphatase (ALP) staining after 7 days' treatment with osteoblast differentiation introduction medium. (F) Primary bone MSCs, isolated from the bilateral femur/tibia from $3 \mathrm{~d}$ or $7 \mathrm{~d}$ after the final injection of saline - or CPD-treatment. Then, primary bone MSCs was cultured with osteoblast differentiation introduction medium for 7 days for ALP staining and 21 days for Alizarin R stains. All the in vitro assays were independently repeated for 3 times or with at least 3 mice. 
viable cells between each CPD working solutions group and control (Supplementary Figure 2). Taken together, these findings indicated that CPD dose-dependently inhibited RANKL-mediated osteoclastogenesis without affecting the viability of BMMs.

\section{CPD inhibited the expression of TRAF-6, NFATc1 and c-Fos in mice or in BMMs}

RANKL binds to its receptor RANK in the membrane of pre-osteoclasts, and recruits members of TRAF family, such as TRAF-3 and TRAF-6 [35], to activate a series of downstream signaling, such as the action of critical transcription factors (NFATc1, c-Fos) that are necessary for osteoclastogenesis [37]. To identify the molecular mechanism involved in the regulation of CPD on osteoclastogenesis, the expression of TRAF-3, TRAF-6, NFATc1 and c-Fos was measured.

As compared to those in control group, the protein expression of TRAF-6, NFATc1 and c-Fos were significantly decreased in the tibia of mice at day 12 after CPD treatment $(P<0.001$, Figure 7A and 7B). However, there was no significant difference in the expression of TRAF-3 and RANK between the two groups (Data not shown).

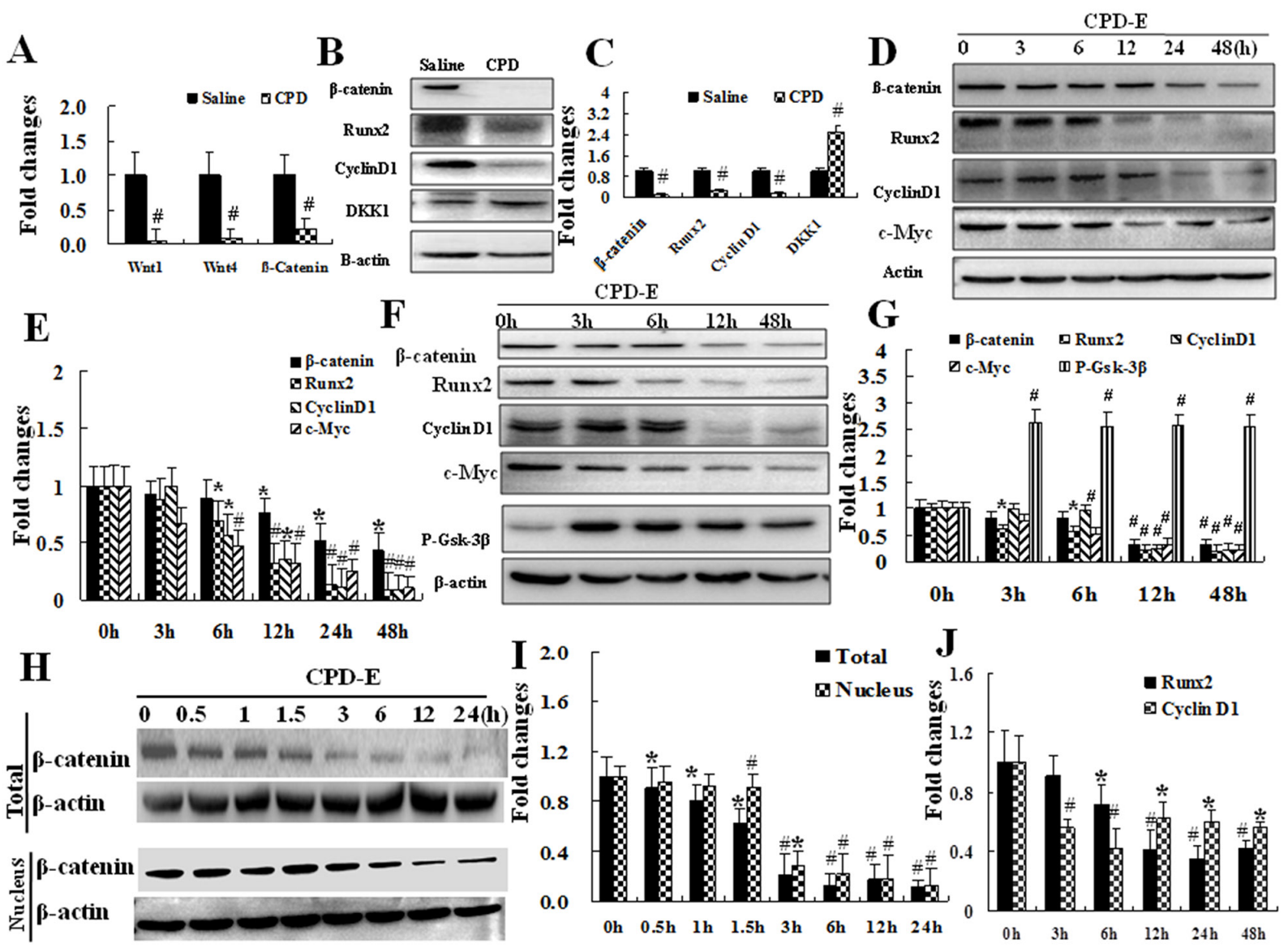

Figure 5: Cyclophosphamide suppressed the activation of Wnt/ß-catenin pathway involved in osteoblastogenesis in mesenchymal stem cells. (A) LV4 5 from saline or CPD- treated mice (7d) were harvested before accessing the mRNA expression of Wnt1, Wnt4, and $\beta$-catenin by real-time PCR. ${ }^{\sharp} P<0.01$, vs. saline group. Primary bone MSCs from saline or CPD-treated mice (7d) were harvested, cultured, and lysed 1days later. The expressions of $\beta$-catenin, Runx2, Cyclin D1and DKK1 were measured with western blot assessments (B) and quantified (C). ${ }^{\#} P<0.01,{ }^{\S}<0.001$ vs. saline group. (D, E) Primary bone MSCs from CPD-treated or saline control were harvested and cultured with CPD-H for 0, 3, 6, 12, 24 and 48h. The protein expressions of $\beta$-catenin, Runx2, Cyclin D1 and c-Myc were assessed with western blot (D) and quantified (E), ${ }^{*} P<0.05,{ }^{*} P<0.01$, vs. 0 hour. (F and G) C3H10T1/2 was cultured with CPDCon or CPD-H for $0 \mathrm{~h}, 3 \mathrm{~h}, 6 \mathrm{~h}, 12 \mathrm{~h}$ and $48 \mathrm{~h}$. The protein expressions of $\beta$-catenin, Runx2, Cyclin D1, c-Myc and P-Gsk-3 $\beta$ were assessed with western blot $\mathbf{( F )}$ and quantified (G). ${ }^{*} P<0.05,{ }^{\#} P<0.01$, vs. 0 hour. (H\&I) Total and nucleus proteins were harvest from C3H10T1/2 and the proteins were harvested at different time $(0,0.5,1,1.5,3,6,12,24 \mathrm{~h})$. The protein expression of $\beta$-catenin was assessed with western blot $\mathbf{( H )}$ and quantified (I). ${ }^{*} P<0.05,{ }^{\#} P<0.01$, vs. 0 hour.C3H10T1/2 cell was treated with CPD-H for different times $(0,3,6$, 12, 24, 48h). The mRNA expression of Runx 2 and Cyclin D1 was assessed with real-time PCR (J). ${ }^{*} P<0.05,{ }^{\#} P<0.01$, vs. 0 hour. For quantification in each assays, Ratios of targeted markers to $\beta$-actin were obtained by dividing the densitomentic reading of these markers with that of $\beta$-actin, and the value calculated at $0 \mathrm{~h}$ was set as 1.00 . The All the in vitro assays were independently repeated for 3 times or with at least 3 mice. 
Furthermore, the suppressive effect of CPD on transcription factors for osteoclastogenesis was further assessed using primary BMMs treated with CPD for 24 $\mathrm{h}$ and $48 \mathrm{~h}$. The protein expression of TRAF-6, NFATc1 and c-Fos was significantly down-regulated at both $24 \mathrm{~h}$ $(P<0.01)$ and $48 \mathrm{~h}(P<0.001)$ as compared to those of CPD-Con group (Figure 7C), while, CPD did not affect the protein expression of RANK and TRAF-3 in BMMs (Figure 7D).

\section{CPD repressed RANKL-activated activation of $\mathrm{NF}-\kappa \mathrm{B}$, while exerting no inhibitory effect on the activation of ERK, JNK and p38}

To further elucidate the molecular mechanism by which CPD inhibits osteoclastogenesis, we next examined the protein expression of key factors involved in RANK signaling pathway, such as NF- $\kappa \mathrm{B}, \mathrm{JNK}$, ERK and p38 [32]. The levels of phosphorylated form of I $\mathrm{B} \alpha, \mathrm{JNK}, \mathrm{ERK}$ and p38 were measured to determine the activation of $N F-\kappa B$, JNK, ERK and p38 signaling pathway by using Western blot analysis (Figure $8 \mathrm{~A}$ and $8 \mathrm{~B}$ ). The results showed that RANKL treatment $(\mathrm{M}+\mathrm{R})$ tremendously increased levels of phosphorylated expression of I $\mathrm{B} \alpha$, JNK, ERK and p38 at 5 minute and 10 minute in BMMs comparing to control without RANKL treatment (M). Phosphorylation of JNK, ERK and p38 were not affected by CPD-Con or CPD-D. However, CPD-D suppressed the phosphorylation of I $\mathrm{B} \alpha$ in contrast to control or CPD-Con at $5 \mathrm{~min}(P<0.05)$ and $10 \min (P<0.01)$, indicating that CPD did not inhibit the activation of JNK, ERK or p38 protein, but suppressed the activation of $\mathrm{NF}-\mathrm{\kappa B}$ in BMMs.

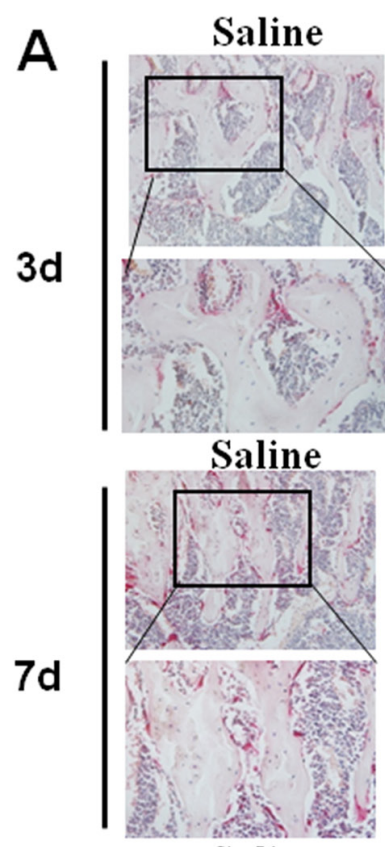

Saline

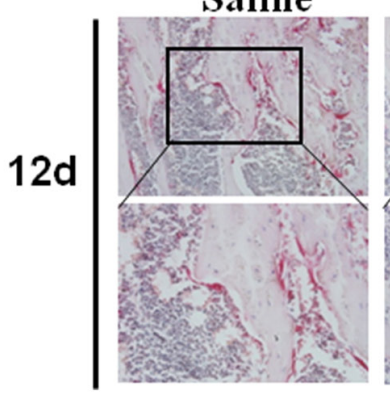

CPD

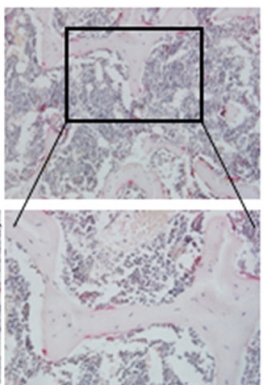

CPD

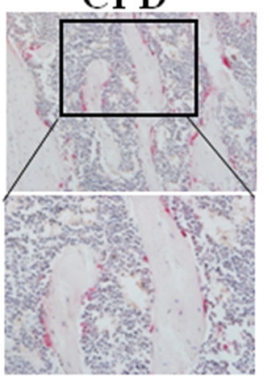

CPD

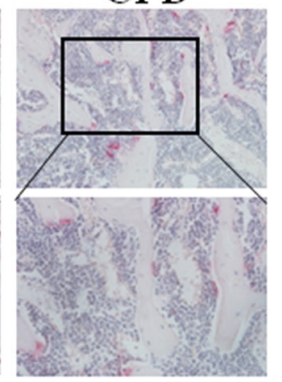

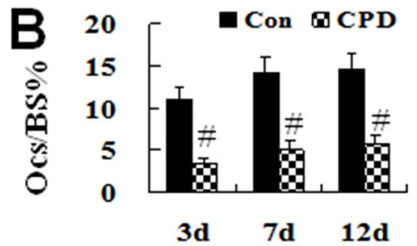
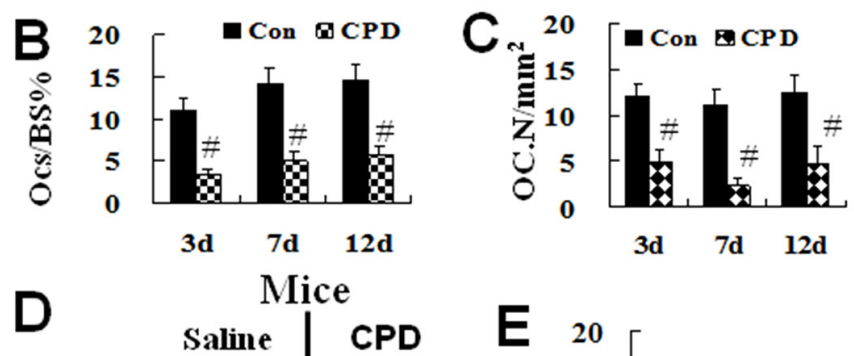

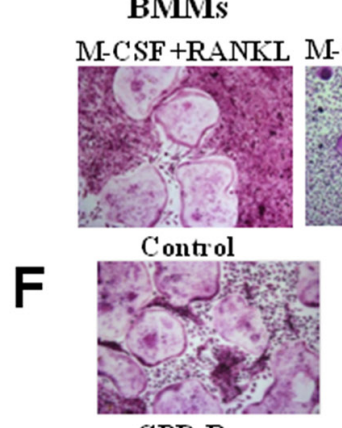

CPD-B

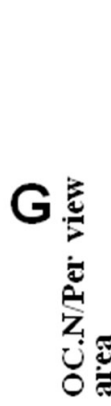

5
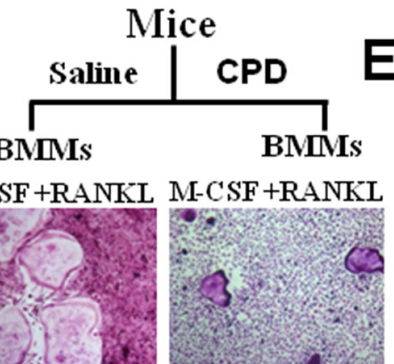

CPD-con

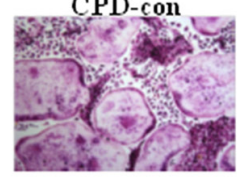

CPD-C
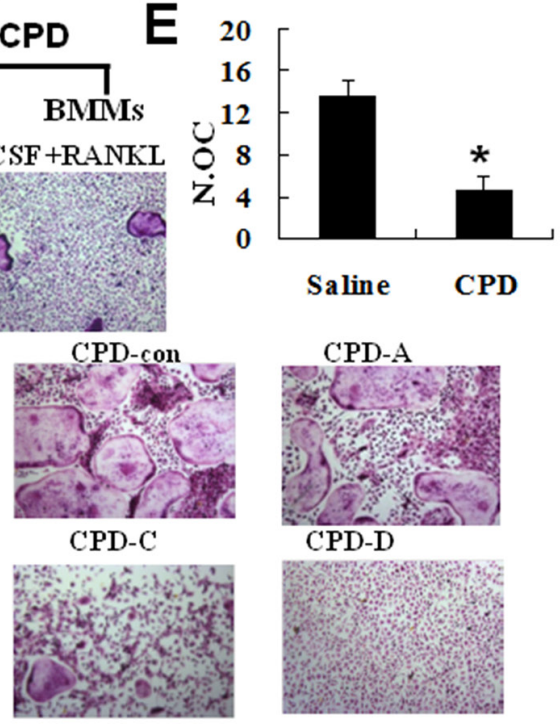

CPD-D
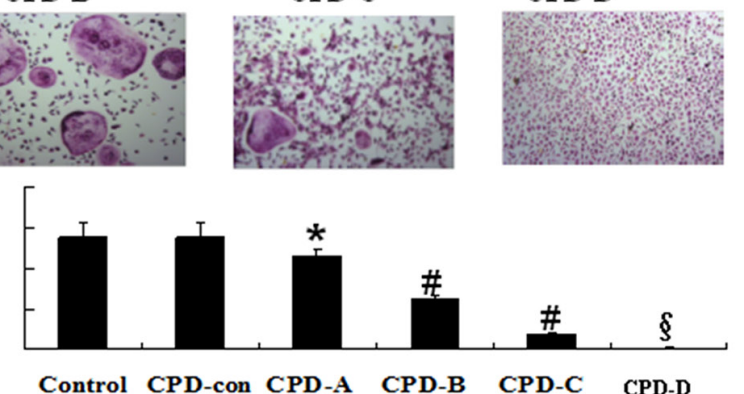

Figure 6: Cyclophosphamide suppressed the activity of TRAP and the capacity of osteoclastogenesis in mice. (A) TRAP staining was performed on femurs at day 3, 7 and 12 after the C57BL/6 mice were injected with saline (Con) or cyclophosphamide (CPD) for 7 consecutive days. (B) Quantification of osteoclasts-covered surface over bone surface. (C) Osteoclasts number. (D) BMMs from saline or CPD-treated mice (12 days) were cultured with M-CSF (44 ng/ml) and RANKL (100 ng/ml) for 5 days before performing TRAP staining. (E) The number of multinucleated TRAP-positive cells ( $>3$ nuclei). ${ }^{*} P<0.01$, vs. saline control. (F) BMMs from C57BL/6 mice were harvested, and treated with M-CSF (44 ng/ml) and RANKL (100 ng/ml) as control, or M-CSF (44 ng/ml) and RANKL (100 ng/ml) plus CPD-Con, CPD-A, CPD-B, CPD-C and CPD-H for 5 days before performing the TRAP staining. The number of osteoclast were quantified (G), ${ }^{*} P<0.05,{ }^{*} P<0.01,{ }^{\S} P<0.001$, vs. saline control. 


\section{CPD inhibited the expression of osteoclastic resorptive factors activated by RANKL}

RANKL up-regulates the differential expression of numerous genes, which are not only requirement for normal osteoclastogenesis but also necessary for bone resorption [46]. Particularly, bone resorption activity was determined by a series of RANKL-stimulated resorptive factors, including MMP9, Car2, Ctsk and TRAP [40], the expression of which were measured in this study. The results demonstrated that the protein expression of MMP9 and Ctsk was significantly down-regulated in CPD-treated group as compared to those in saline control group $(P<0.05$, Figure 9A and 9B).

To further confirm the inhibitory effect of CPD on RANKL-activated genes in osteoclastogenesis, we next performed in vitro investigations to assess mRNA levels of MMP9, Car2, Ctsk, and TRAP with CPD-D in BMMs. As shown in Figure 9C and 9D, RANKL significantly activated the gene expression of MMP9, Ctsk, TRAP and Car2 at 24,48 or $96 \mathrm{~h}$ in BMMs. However, CPD-D significantly suppressed RANKL-induced expression of MMP9, Ctsk, TRAP and Car2 genes at 24, 48, and $96 \mathrm{~h}$ in BMMs. Our results demonstrated that CPD significantly inhibits RANKL-mediated expression of MMP9, Ctsk, TRAP, and Car2 genes in the process of osteoclast formation. Taken together, these findings indicated that CPD exerts inhibitory effects on RANKL-induced expression of osteoclast genes encoding MMP9, Ctsk, TRAP and Car2.

\section{DISCUSSION}

This is the first study to elucidate the roles of CPD in osteoblastogenesis and osteoclastogenesis using both in vitro and in vivo assays. Our study found that CPD
A
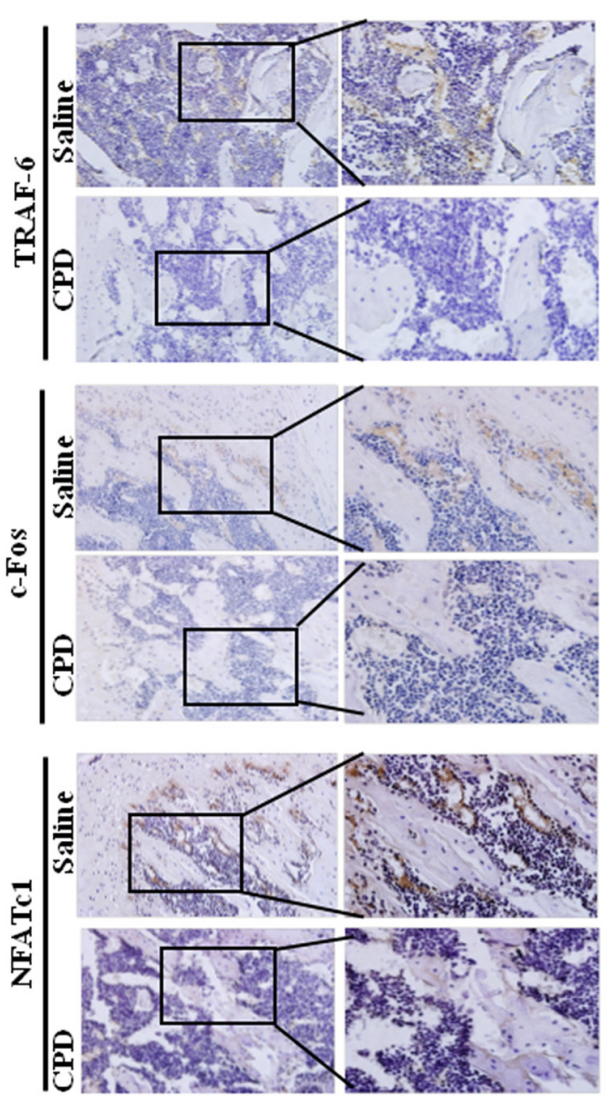
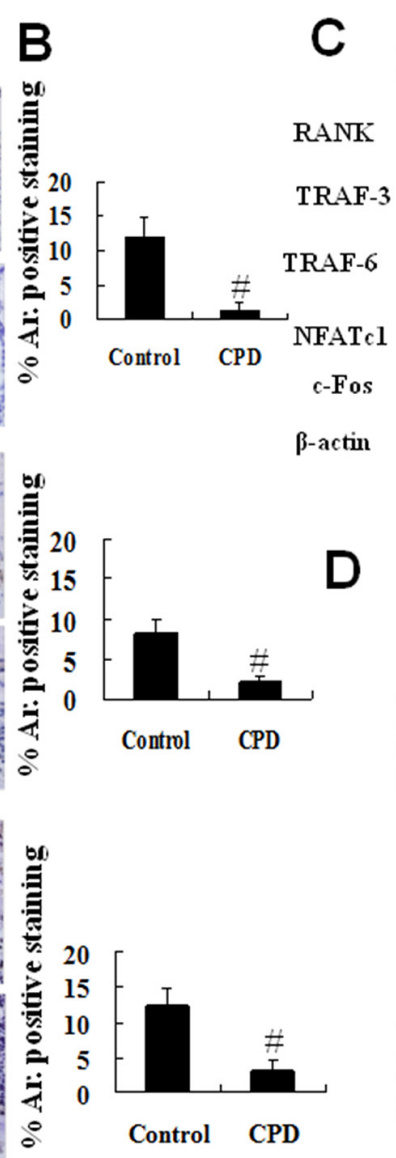

D

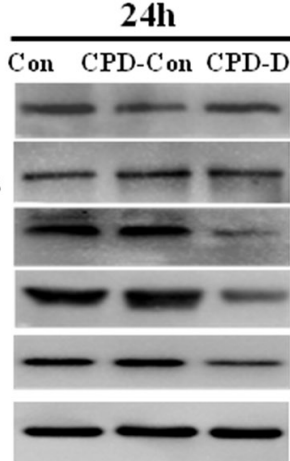

$48 h$

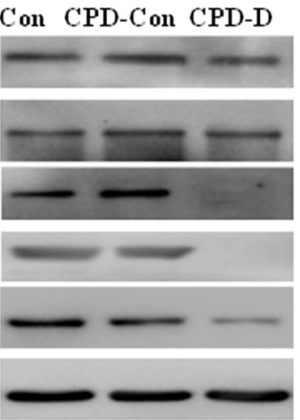

24h

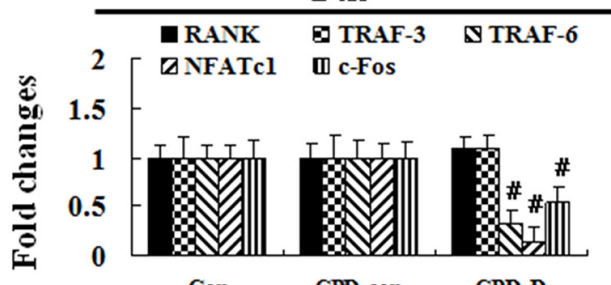

Con

CPD-con CPD-D

$48 h$

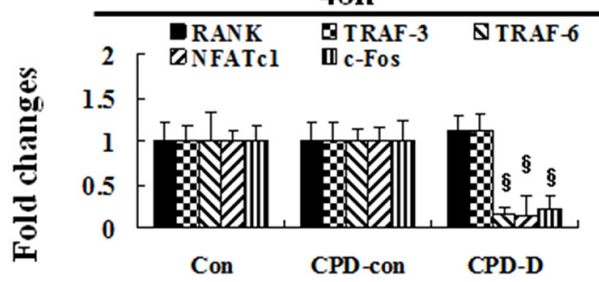

Figure 7: Cyclophosphamide caused the decreased expression of TRAF-6, c-Fos, NFATc1 in mice or in BMMs. (A) 4-6-week-old male C57BL/6 mice were administered with saline or cyclophosphamide (CPD, $100 \mathrm{mg} / \mathrm{kg} /$ day) for 7 consecutive days by intraperitoneal injections. Mice were then maintained for additional 12 days before harvesting. The protein expression of TRAF-6, NFATc1 and c-Fos in tibia were assessed by immunohistochemistry. One set of representative images $(20 \times$ and $40 \times)$ were shown, and the percentage (\%) of the positive staining area to the whole view area were quantified (B). ${ }^{\#} P<0.01$, vs. saline control. (C) BMMs were treated with M-CSF (44 ng $/ \mathrm{ml}$, Con), or plus CPD-Con or CPD-D for $24 \mathrm{~h}$ or $48 \mathrm{~h}$. The protein expression of RANK, TRAF-3, TRAF-6, NFATc1 and c-Fos were measured. (D) Ratios of RANK, TRAF-3, TRAF-6, NFATc1 and c-Fos to $\beta$-actin were obtained by dividing the densitomentic reading of RANK, TRAF-3, TRAF-6, NFATc1, and c-Fos with that of $\beta$-actin, and the value calculated for con at $24 \mathrm{~h}$ was set as 1.00 . The protein bands were quantified. ${ }^{\sharp} P<0.01,{ }^{\S} P<0.001$ vs. control. 
treatment induced significant deteriorations of bones in mice. On one hand, CPD exerted the inhibitory effects on osteoblastogenesis through suppressing the Wnt/ $\beta$-catenin signaling shown by the decreased expression of $\beta$-catenin, Wnt 1, Wnt10b and Cyclin D1 and the increased expression of DKK1. Consequently, CPD decreased the expression of osteoblast-specific factors like Runx2, Osteocalcin, ALP and the number of bone MSCs (Figure 10A). On the other hand, CPD inhibited the activity of osteoclast as demonstrated by the down-regulation of MMP9, Ctsk, TRAP and Car2. Moreover, RANKL-mediated osteoclastogenesis was also suppressed by CPD, which down-regulated the expression of TRAF-6, NFATc1, NF- $\kappa$ B and c-Fos (Figure 10B). Finally, the serum level of PINP, one of the bone formation markers, and $\beta$-CTX, one of the bone resorption markers were both decreased in CPDtreated mice (Data not shown). This study has provided new insights into the roles of CPD in bone remodeling, especially for understanding the underlying mechanism for bone loss of CPD-treated patients.

CPD, one member of alkylating agent family, was artificially synthesized in 1958 and prescribed in clinical practice from 1970s for treating a series of diseases [47], including cancers, immunological diseases, and rheumatoid
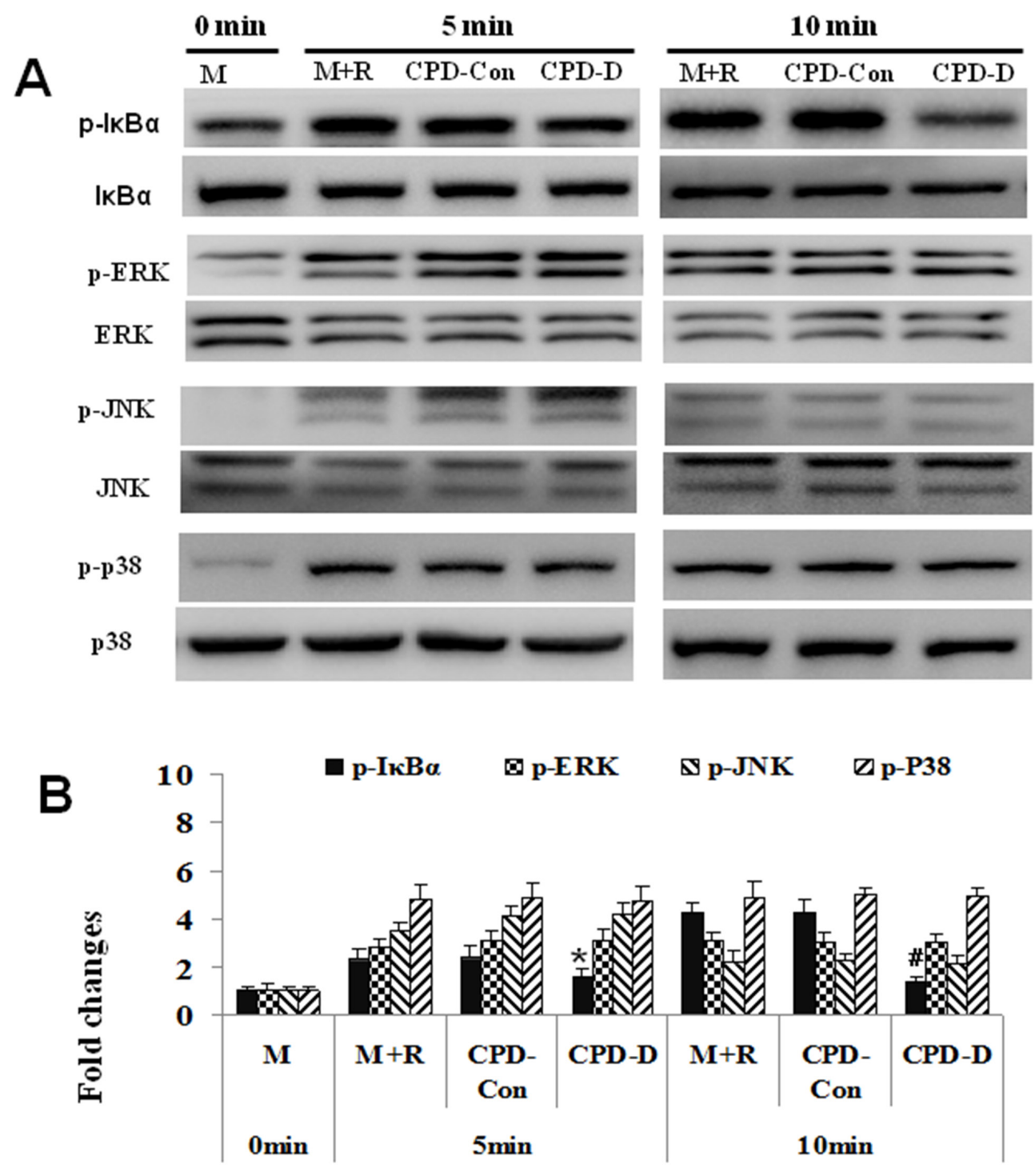

Figure 8: CPD repressed RANKL-activated expressions of NF-кB, while exerting no inhibitory effect on the expressions of ERK, JNK and p38. (A) BMMs were cultured with M-CSF (44 ng/ml) alone (M), M-CSF (44 ng/ml) and RANKL (100 ng/ml) $(\mathrm{M}+\mathrm{R}), \mathrm{M}+\mathrm{R}$ plus CPD-Con, or CPD-D for 5 or $10 \mathrm{~min}$. The protein expression of non-phosphorylated and phosphorylated form of I $\mathrm{B} \alpha$, ERK, JNK, and p38 was determined. Ratios of the phosphorylated form to the total protein were obtained by dividing the densitometric reading of the phosphorylated form with that of the total proteins in each group, and 0 min was set as 1.00 , and quantified (B), ${ }^{*} P<0.05$, ${ }^{\#} P<0.01$, vs. control or 0 min. 
arthritis. CPD exerts anti-tumors effect by suppressing the proliferation of tumor cells via inhibiting the replication of DNA [48] and serves as one of the most widely used chemotherapy drugs. The prescriptions of CPD regiments have greatly increased overall survival rate of cancer patients [49]. However, there are also side effects associated with CPD usages, such as myelosupression [20], cognitive dysfunction [50] and others [51]. The damage of CPD on bone was first reported in 1982, which indicated that the local administrations of CPD inhibited the mandibular growth and induced bone destructions in rats [52]. The accumulating clinical evidences has shown that the usages of CPD and its regiments caused osteoporotic phenotype in patients with breast cancer [10, 11, 53, 54]. Moreover, recent studies have indicated that the treatment of CPD in patients appear to suppress the expression of interleukin (IL)-6 [55], one bone resorption marker, and inhibit the osteoclasts-covered bone surface $[6,43]$. Moreover, low dosage of metronomic CPD alleviated breast cancerinduced osteolysis [56]. Interestingly, the application of CPD caused bone loss but meanwhile increased the higher level of Osteocalcin and OPG in breast cancer patients [12]. Taken together, these studies indicates CPD may exert inhibits bone resorption by inhibits the activity of osteoclast. This is also our propose to address the biological influences and mechanism of CPD on bone, particular on osteoclast, are far more complicated than we have realized.

Previous clinical finding indicated that the application of CPD induced early onset of menopausal in women as well as bone loss in female mice [8]. Herein we studied the effects of CPD on bone biology of male mice. This study showed that CPD treatment induced the phenotype of bone loss in male mice, meanwhile, subsequently FACS assays in our study with CPD-treatment mice indicated that CPD also caused the decrease of MSC numbers. The reduced number of MSCs is one biological manifestation of CPD-induced
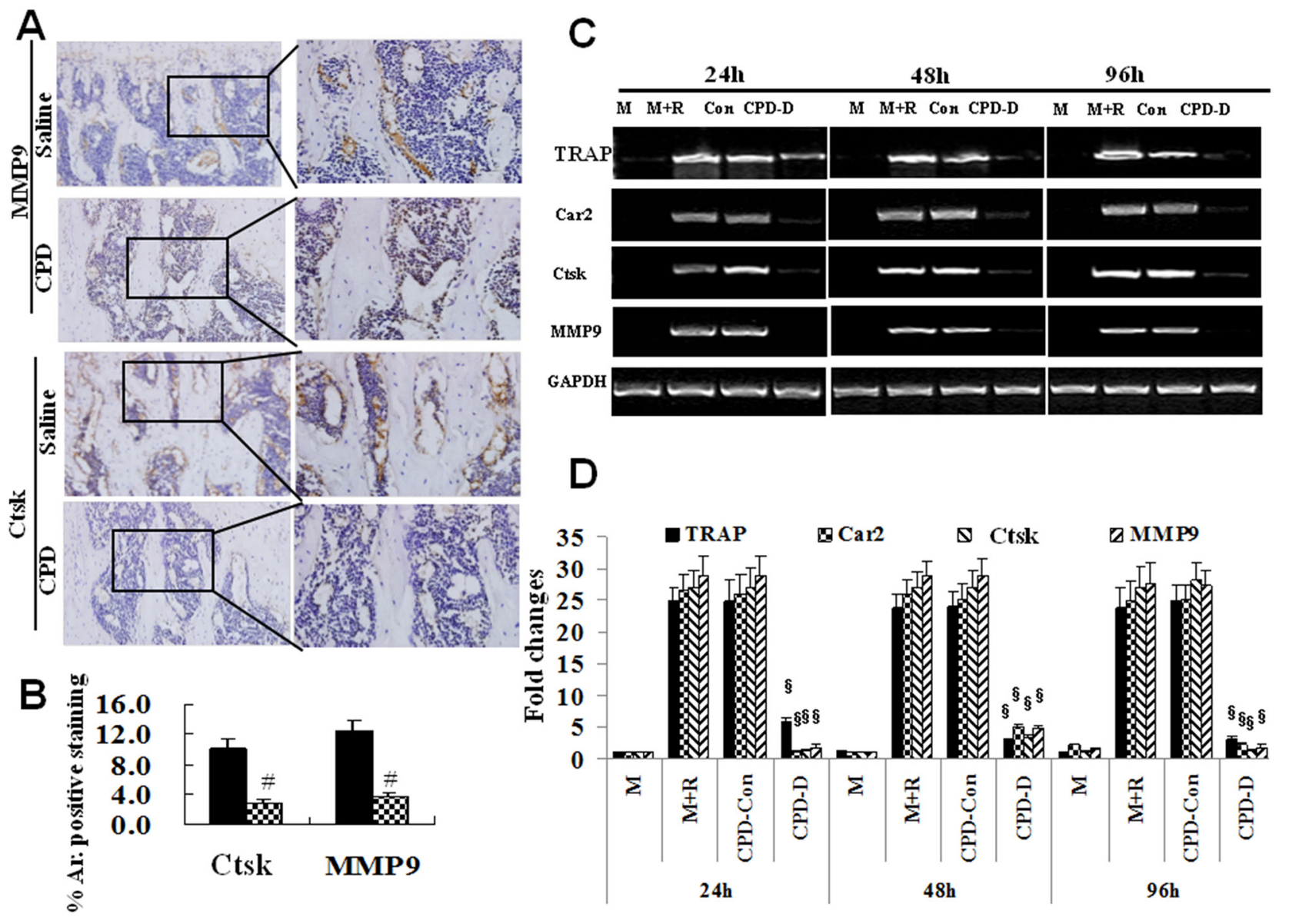

Figure 9: Cyclophosphamide caused the depressed expression of Ctsk and MMP9 in mice and Ctsk, MMP9, TRAP and Car II in BMMs. (A) 4-6-week-old male C57BL/6 mice were administered with saline or cyclophosphamide (CPD, 100 mg/kg/ day) for 7 consecutive days by intraperitoneal injections. The protein expression of Ctsk and MMP9 in tibia of mice at day 12 after CPD treatment was assessed. One set of representative images $(20 \times$ and $40 \times)$ under each condition were shown, and the percentage $(\%)$ of the positive staining area to the whole view area were quantified (B) ${ }^{\#} P<0.01$, vs. Saline control. (C) BMMs were treated with M-CSF (44 ng/ $\mathrm{ml}, \mathrm{M}$ ), or combined with RANKL (100 ng/ml, M+R), or M+R plus CPD-Con, or CPD-D for 24h, 48h and 96h prior to isolation of total RNAs for semi quantitative RT-PCR analysis. (D) Quantification of the mRNA expression of TRAP, Car2, Ctsk and MMP9, ${ }^{\S} P<0.01$, vs. CPD-Con. 
microenvironment for bone metastasis [57], which is in accordance with the previous findings indicating that CPDinduced microenvironment promoted subcutaneous tumor growth and skeletal metastasis in animal models [58, 59].

CPD induces the expressions of several factors, such as C-C chemokine ligand (CCL) 2, IL-6, and VEGF-A, in bone marrow to create a pro-metastatic microenvironment for cancer cells and osteoclast to survive and the suppressed expression of MSCs in bone marrow. Therefore, the following bone mineral density (BMD) decreases are associated because of the deficiency of MSCs and accelerating osteoclast-induced bone loss that caused by bone metastasis [60]. The FACS result in this study indicated that CPD-treatment induced decreased numbers of MSCs in mice, which may contribute to the loss of bone mass. The decrease of osteoblastogenesis was further identified by ALP staining. CPD-induced decrease of bone formation and osteoblast activity were demonstrated by the down-regulation of the expression in osteoblastic differentiated factors, such as Runx2, Osteocalcin, col-1, Wnt 1, Wnt 4, Wnt10b and Cyclin D1 and the up-regulation of the expression of DKK1 in primary bone MSCs.

Previous investigations have indicated that CPD induced more adipogenesis rather that osteoblastogenesis by altering the switch of MSCs differentiation [21]. Studies have demonstrated that the over-activity of Wnt/ $\beta$-catenin in MSCs changed the balance of adipocyte/ osteoblast by inducing the osteogenesis transcription factors but inhibiting the adipogenesis transcriptions factors PPAR $\gamma$, thus, induced more osteogenesis (ALP) and bone formation [61]. Therefore, the down-regulated expression of $\mathrm{Wnt} / \beta$-catenin may account for CPDinduced bone loss because of the promotion of adipocytes instead of osteoblasts besides the direct inhibitory effects on osteoblast-mediated bone formation [13, 21]. This study indicated that CPD induced bone loss in bone remodeling by decreasing the number of MSCs, inhibiting osteoblastogenesis, and contributing to the suppressed bone formation and bone loss.

On the other hand, the molecular mechanism of CPD in RANKL-mediated osteoclastogenesis need to be further addressed. Although previous CPD study demonstrated that regiments CEF enhanced bone resorption [8, 54], CPD intervention suppressed bone resorption marker, such as IL-6 [55], and exerted bone protective effects on breast cancer-induced osteolysis [56]. M-CSF and RANKL are two necessary cytokines for osteoclastogenesis of BMMs [62]. M-CSF mainly promotes the survive and proliferation of BMMs, while RANKL drives BMMs into the osteocalst lineages commitment [34] and RANKL-activated signaling plays critical roles for the differentiation, survival, activity of osteoclast and consequently bone resorption [63, 64]. Therefore, the identification of the effects of CPD on RANKL-activated signaling will aid us to understand the systematic roles of CPD on osteoclastogenesis and bone remodeling. This study demonstrated the inhibitory effects of CPD on osteoclastogenesis, and the potential mechanism may be attributed to its suppressive effects of CPD on the expression of TRAF-6, NFATc1, NF-kB and c-Fos which are involved in RANKL-mediated osteoclastogenesis [38]. Meanwhile, CPD suppressed bone resorption by inhibiting osteoclast genes encoding MMP9, Ctsk, TRAP and Car2. The similar bone-protective effects of chemotherapy agents was identified by using one 2-methosyestradiol analogue ENMD-1198, which inhibited osteolysis in breast cancerinduced mice and exerted more pronounced bone-protective effects than low-dose metronomic CPD in mice model [56].

The inhibition of CPD on osteoclastogenesis was not in accordance with the findings that CPD led to osteoporosis phenotype in this study. Similar result was reported that thiazolidinedione (TZD), targeting PPAR $\gamma$, exerted the inhibitory effects on both osteoclastogenesis and osteoblastogenesis, and finally induced bone loss and increased higher risk of fracture in patients with type 2 diabetes $[65,66]$. As we all know, normal bone marrow is one complex microenvironment that houses hematopoietic stem cells (HSCs) and MSCs to maintain the balance of bone formation and bone resorption [45, 67]. It was supposed that even the inhibitory effect of CPD on bone resorption can partly compensate for bone loss mainly caused by the influences of CPD on MSCs and osteoblastogenesis in this study, it cannot fully keep up with the rapid bone loss. Hereby, our study potentially indicated that CPD inhibited bone formation and bone resorption in favor of bone formation. Therefore, the detailed regulatory mechanism of CPD on bone remodeling still need to be further investigated.

For clinical practice, this study indicates that the bone formation plays crucial roles in preventing CPD-induced bone loss. The treatment for bone loss induced by marketed drugs in treating chemotherapy are mainly targeting osteoclast to inhibit bone resorption [68], but the clinical effect is not good as expected. Our findings demonstrated that targeting osteoblasts to improve osteoblastogenesis might be one effective approach, and the novel biochemical agents, stimulating osteoblastogenesis and suppressing adipogenesis, may exert better bone-protective effects in rescuing CPD-induced bone loss.

The usages of CPD have been reported to cause side effects on a number of organs. Studies have reported that CPD caused hepatoxicity, nephrotoxicity, reprotoxicity, cardiotoxicity, and myelosuppression [69-71], which may account for bone loss by their indiect effects on bone:1). The reprotoxicity accelerates bone loss by decreasing the sexual hormones such as estrogen and androgen [10]; 2). The nephrotoxicity lead to the disorder of the metabolism of calcium and phosphorus and consequently bone loss. But the direct effects of CPD on bone remodeling are still not fully understood. Our study first established the direct effects of CPD on bone remodeling by both affecting osteoblastogenesis and osteoclastogenesis. Furthermore, our study also support that CPD caused myelosuppression 
as shown by decreased the number of bone MSCs in CPD treated mice (Figure 4A and 4B). At the molecular level, the inhibitory effect of CPD is similar to the inhibitory effects of methotrexate, one chemotherapy agent, that exert on the expression on $\mathrm{Wnt} / \beta$-catenin signaling in bone [72].

The biological influences of CPD on bones have closely relationship with the dosages, and frequency of the interventions while the proper selection for CPD can produce less toxicity/less side effects for both animal and in vitro investigation. Normally, the severity of toxicity to normal cells in humans and experimental animals is increased with the high dose of CPD or $\mathrm{CPD}$ regiments. According to the dosages in clinical application, the dosage of CPD and CPD regiment ranged from $10 \mathrm{mg} / \mathrm{kg}$ to $350 \mathrm{mg} / \mathrm{kg}$ but with different cycles of chemotherapy treatment. One study induced bone disorders by intravenous tail injections of CPD regiment $(10 \mathrm{mg} / \mathrm{kg}$ ) for six cycles (weeks) in SpragueDawley rats [18], indicating that the low dosages of CPD also cause toxicity on bone tissue. Moreover, commercial CPD was administrated $(50 \mathrm{mg} / \mathrm{kg}$ ) with intraperitoneally to Swiss albino mice to induce the hypercellularity in bone marrow and spleen [73]. Furthermore, a single dosage of CPD $(150 \mathrm{mg} / \mathrm{kg})$ induced rapid and severe hematopoietic suppression in male Ku Ming (KM) mice [20] and same dosages of CPD were performed to induce the myelosupression and bone osteoporosis phenotypes in $\mathrm{C} 57 \mathrm{BL} / 6$ mice [74]. In addition, the intraperitoneal injection with the one single dosage of CPD (200mg/ $\mathrm{kg}$ ) has been reported to cause the myelosupression in male BALB mice [75]. To our knowledge, the highest dosage $(350 \mathrm{mg} / \mathrm{kg}$, i.p) intervention with of CPD has been identified to enhance experimental prostate cancer metastasis [76]. In our study, we treated the mice with $100 \mathrm{mg} / \mathrm{kg}$ of CPD at 7 seven consecutive interventions. Our intervention was able to induce commonly seen side effects, such as myelosupression, based on the previous animal study and thereafter to investigate their effects on bone loss in C57BL/6 male mice. However, the dosage and intervention time was performed at one rather moderate level but not at lethally dosage. Meanwhile, CPD at $2000 \mu \mathrm{M}$ (CPD-D) could effectively inhibit osteoclastogenesis (Figure 6F and 6G) but exerted no significant effects on cell viability of BMMs (Supplementary Figure 2). Therefore, these data indicated that the osteoclastogenesis inhibited by CPD was not due to the toxicity of CPD on BMMs.

However, there are still several limitations in our studies. First, our study was performed with male mice and the exact effects of CPD on female have not been investigated based on our preliminary finding indicates that the male mice were more sensitive than female for CPD intervention [77]. Therefore, future work will be performed to address this issue. Second, CPD are commonly prescribed with regiments, therefore, it is necessary to carry out more investigations on CPD regiments. Third, the intervention time with $\mathrm{CPD}$ is short (7 days). Finally, only one dosage was performed in this

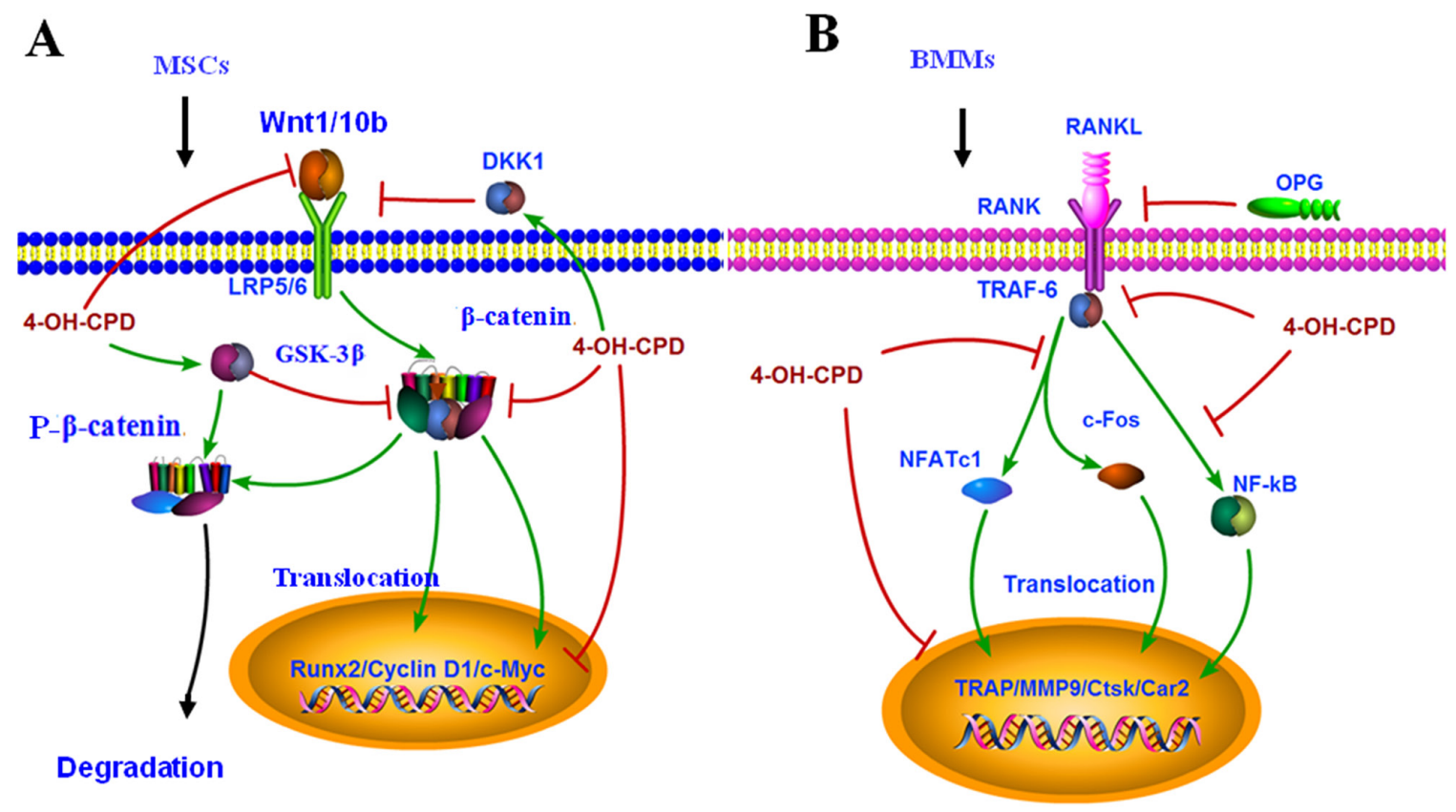

Figure 10: Molecular mechanism of cyclophosphamide in inhibiting osteoblastogensis and osteoclastogenesis. (A) Cyclophosphamide (CPD) inhibits osteoblastogenesis by suppressing Wnt/ $\beta$-catenin activated Cyclin D1, Runx2, c-Myc and stimulating the expression of GSK-3 $\beta$ and DKK1. (B) CPD inhibits osteoclastogenesis by suppressing RANKL-activated expression of TRAF-6, NFATc1, NF-кB, с-Fos and osteoclast encoding genes activity of MMP9, Ctsk, TRAP and Car2. 
Table 1: The sequences of primers used in this study

ß-actin:

forward,5'-CCTGTACGCCAACACAGTGC-3'; reverse, 5' - ATACTCCTGCTTGCTGATCC-3';

Oc:

forward, 5' - CTTGAAGACCGCCTACAAAC-3'; reverse, 5'- GCTGCTGTGACATCCATAC-3';

ALP:

forward, 5' - GAATCAAATGTTCAGGGTGGT-3'; reverse, 5' - TGGCACGTTAAAGGTAATCAG-3';

Runx2:

forward,5'-CTCTTCTGGAGCCGTTTATGT-3'; reverse, 5'- GTTTCTTAGGGTCTTGGAGTGA-3'; Wnt1:

forward, 5' - ACAGCGTTCATCTTCGCAATCACC-3'; reverse,5'-AAATCGATGTTGTCACTGCAGCCC-3';

Wnt4:

forward, 5' - CTCAAAGGCCTGATCCAGAG-3'; reverse,5'- GTCCCTTGTGTCACCACCTT-3'; Wnt10b:

forward, 5'- TAACCACGACATGGACTTCGG-3'; reverse,5'-TCCGCTTCAGGTTTTCCGTTA-3’; Cyclin D1:

forward,5'-CTCCGTATCTTACTTCAAGTGCG-3'; reverse, 5'- CTTCTCGGCAGTCAAGGGAA-3';

ß-Catenin:

forward,5'-CACGCAAGAGCAAGTAGCTG-3'; reverse,5'-TCTGTGATGGTTCAGCCAAG-3';

in vivo study and the dose-dependent effect of should be studied. Taken together, the dosage, and frequency is vary to the clinical practice, and will limit the usages in the management of patient.

In summary, our animal and in vitro studies have demonstrated the following points: 1) CPD treatment induced the osteoporotic phenotype in mice associated with the decrease of bone formation and resorption. 2) CPD exerted inhibitory effects on osteoblastogenesis by suppressing Wnt $/ \beta$-catenin signaling. 3) CPD exerted inhibitory effects on RANKL-mediated osteoclastogenesis by suppressing RANKL-activated signaling. 4) The changes of bone formation, other than bone resorption, played critical roles in CPD-induced bone loss.

\section{MATERIALS AND METHODS}

\section{Reagents}

Cyclophosphamide (CPD) was purchased from Shengdi Company (Jiangsu, China). M-CSF and RANKL were purchased from Peprotech (New Jersey, USA). Primary antibodies against active $\beta$-catenin, Runx2, Cyclin D1, c-Myc, I $\mathrm{B} \alpha$, phospho-I $\mathrm{B} \alpha$, JNK, phosphoJNK, p38 and phospho-p38 were purchased from Cell Signaling Technology (Beverly, MA, USA) and others against TRAF-6, TRAF-3, NFATc1, RANK and c-Fos were purchased from Santa Cruz Biotechnology (CA, USA). The antibodies against $\beta$-catenin, phospho-Gsk$3 \beta$, and DKK1 were purchased from Abcam Company (Cambridge, UK).

\section{Animal treatments}

Six-week-old male C57BL/6 mice (SLAC Laboratory Animal Co. Ltd., Shanghai, China) were maintained in animal center of Longhua hospital with light: dark (12h:12h) condition and fed with commercial diet and distilled water ad libitum during experimental period. The mice were administrated with CPD (100mg/ $\mathrm{kg}$ /day) by intraperitoneal injection for 7 consecutive days and subsequently maintained for additional 3, 7 , and $12 \mathrm{ds}$. For each time points, tibia and lumbar vertebra 1 3 were collected for histological analysis and lumbar vertebra 4 5 were harvested for RNA analysis. All of the experimental protocols were performed with the approval of Institutional Animal Care and Use Committee of the Shanghai University of Traditional Chinese Medicine, and conformed to the National Institutes of Health Guide for Care and Use of Laboratory Animals (Publication No. 8523, revised 1985).

\section{$\mu \mathrm{CT}$ analysis}

Tibia and LV1 3 were fixed in 10\% Neutral Buffered Formalin (Wexis, Guangzhou, China) for 24h, washed for 2 hours with tap water, and examined. The samples in each group were scanned to develop threedimensional (3D) mode. The analyses were carried out using $\mu$ CT80 radiograph microtomograph (Scanco Medical AG, Switzerland), associated with $3 \mathrm{D}$ reconstruction by $\mu \mathrm{CT}$ Ray V3.4A model visualization software (Scanco Medical AG, Switzerland). The following quantitative parameters including bone mineral density (BMD), ratio of bone volume to tissue volume (BV/TV), trabecular bone numbers (Tb.N), trabecular bone thickness (Tb.Th), and trabecular separation (Tb.Sp), were obtained.

\section{HE staining}

Tibia and lumbar vertebra $1 \sim 3$ were fixed in $4 \%$ paraformaldehyde for $24 \mathrm{hs}$, decalcified in 10\% EDTA for 
4 weeks, and embedded in paraffin wax. The sections were stained with hematoxylin and eosin (H\&E). Subsequently, the representative images were obtained with auto capture system (Olympus BX50, Tokyo, Japan).

\section{Immunohistochemistry analysis}

Tibia sections $(5 \mu \mathrm{m})$ were deparaffinized, rehydrated with PBS ( $\mathrm{pH} 7.4$ ), treated with aqueous $3 \% \mathrm{H}_{2} \mathrm{O}_{2}$ for 10 $\mathrm{min}$, and antigen was retrieved with $0.1 \%$ trypsin $(\mathrm{M} / \mathrm{V})$ in $0.1 \% \mathrm{CaCl}_{2}$ at $37^{\circ} \mathrm{C}$ for $10 \mathrm{~min}$. The sections were blocked with $5 \% \mathrm{BSA}$ at RT for $30 \mathrm{~min}$, then the antibodies for active $\beta$-catenin, Runx2, Osteocalcin, TRAF-6, NFATc1, c-Fos, MMP9, and Ctsk were applied at the concentration of 1:200 and incubated overnight at $4^{\circ} \mathrm{C}$. The slides were incubated with second antibody conjugated HRP for 15 minutes after washing with PBS. Diaminobenzidine was applied for 5 minutes, and slides were then counterstained with H\&E, dehydrated, and mounted with cytoseal mounting medium. The images were obtained by the imagine auto analysis system (Olympus BX50). Bone morphometric analysis was performed with an image autoanalysis system (Olympus BX50, Tokyo, Japan)

\section{Flow cytometry analysis}

Flow cytometry antibodies anti-CD29-FITC, and CompBeads were purchased from BD biosciences (New Jersey, USA). Anti-CD45-PerCPcy 5.5 was from Biolegend (USA) and anti-CD44-APC was from eBioscence (SanDiego, CA, USA). Bone marrow cells from tibia and femur of 10-12-week-old saline or CPD-treated (7 consective days, dosages) male mice were flushed out with PBS plus 2.5\% Fetal bove serum (FBS), filtered, centrifuged, removed the red blood cell, and collected. The final concentrations were $1 \times 10^{7} / \mathrm{ml}$ before performing flow cytometry assays. The negative expression of CD45-PerCPcy5.5, and positive expression of CD29-FITC, and CD44-APC were obtained and analyzed with BD FACS Aria III (New Jersey, USA), and one representative assay was shown.

\section{CM-CPD working solution preparation and identification}

CPD working solution study was prepared with conditioned medium (CM) for in vitro investigations as described previously [78]. Briefly, the mouse hepatocyte BNLCL. 2 cells (Stem Cell Bank from Chinese Academy of Sciences, Shanghai, China) were cultured in $\alpha$-MEM containing $10 \%$ FBS and $1 \%$ P-S to $70 \%$ confluent before starving in $\alpha$-MEM with $1 \% \mathrm{P}-\mathrm{S}$ for $12 \mathrm{~h}$. CPD with the concentrations of $4000 \mu \mathrm{M}$ was added to the $\alpha$-MEM containing $2 \% \mathrm{FBS}, 1 \% \mathrm{P}-\mathrm{S}$ and subsequently cultured for $24 \mathrm{~h}$. The supernatants were centrifuged and collected before filtering with $0.22 \mu \mathrm{m}$ filter unit and are prepared as the primary CM-CPD working solution.
To identify the efficacy and quality of CM-CPD working solution, the liquid chromatography mass spectrometry (LC-MS/MS) analysis were performed. The standard curve (nanogram-peak area) was made with 2 time's serial dilution of 4-Hydroperoxy CPD (from 109ng) which easily converted to 4-OH-CPD. In brief, $10 \mu \mathrm{L}$ standard dilution (plus $190 \mu \mathrm{L}$ CPD-Control), or $200 \mu \mathrm{L} \mathrm{CPD}(4000 \mu \mathrm{M})$ was mixed immediately with $20 \mu \mathrm{L}$ of semicarbazide (SCZ; $2 \mathrm{M}$ ) to derivative $4-\mathrm{OH}-$ $\mathrm{CPD}$ into 4-OH-CPD-SCZ and was vortexed before placing in a water-bath at $35^{\circ} \mathrm{C}$ for $2 \mathrm{~h}$. Next, $100 \mu \mathrm{L}$ acetonitrile was centrifuged, and the supernatant was extracted twice with ethyl acetate. The organic phase was evaporated to dryness and reconstituted with mobile phase (water-acetonitrile, 50:50, and $\mathrm{vol} / \mathrm{vol}$ ). The LC separation was performed on a Thermo Scientific Hypersil Gold column with water-acetonitrile (90:10, $\mathrm{vol} / \mathrm{vol}$ ) as the starting gradient, at a flow-rate of $0.3 \mathrm{~mL} /$ min with a total run time of $15 \mathrm{~min}$. Mass transitions were monitored at $\mathrm{m} / \mathrm{z}: 334 \rightarrow 221$ for $4-\mathrm{OH}-\mathrm{CPD}-\mathrm{SCZ}$ under the positive multiple reaction monitoring (MRM) mode. The curves in consistence of 4-OH-CPD-SCZ are regarded as meet the quality control and selected to perform in vitro study (Figure 1).

Different concentrations of CM-CPD working solutions were prepared with proper dilutions of the primary CM-CPD $(4000 \mu \mathrm{M})$ working solution. For convenience, the working solutions with $\mathrm{CPD}$ at the concentration of $0 \mu \mathrm{M}, 500 \mu \mathrm{M}, 1000 \mu \mathrm{M}, 1500 \mu \mathrm{M}$, $2000 \mu \mathrm{M}, 2500 \mu \mathrm{M}, 3000 \mu \mathrm{M}, 3500 \mu \mathrm{M}, 4000 \mu \mathrm{M}$ were marked as CPD-Control (CPD-Con), CPD-A, CPD-B, CPD-C, CPD-D, CPD-E, CPD-F, CPD-G, and CPD-H respectively, through the whole in vitro study.

\section{ALP and alizarin red staining}

For osteoblast differentiation assays, the primary mesenchymal stem cells (MSCs) were isolated from the bilateral femur and tibia of naïve, saline control or CPD-treated $(100 \mathrm{mg} / \mathrm{kg} /$ day $)$ male C57BL $/ 6$ mice. Bone marrow cavity was flushed with $\alpha$-MEM (Hyclone laboratories, Utah, USA) containing 10\% fetal bovine serum (FBS, Gibco, Carlsbad, CA, USA) and $1 \%$ penicillin-streptomycin. MSCs were cultured with osteoblast differentiation introduction medium $(\alpha$-MEM complete medium containing $10 \mathrm{mmol} / \mathrm{L}$ $\beta$-Glycerophosphate (Sigma), 50mg/L L-ascorbic acid (Sigma), $10^{-8} \mathrm{~mol} / \mathrm{L}$ Dexamethasone (Sigma)) for $7 \mathrm{ds}$ with media refreshed twice weekly. Then cells were fixed for 20min with 10\% Neutral Buffered Formalin followed by washing twice with PBS. ALP staining reagent 1-Step NBT-BCIP (Pierce, Rockford, IL, USA) were added to wells of 24 -well plate for $0.5-1 \mathrm{~h}$ at $37^{\circ} \mathrm{C}$ and the positive staining was shown by Lyons blue. MSCs from naïve mice were cultured with CPD-Con, CPD-B, CPD-D, and CPD-H for 3 days before cultured in the 
osteoblast differentiation introduction medium for ALP staining or Alizarin red staining. To examine the capacity of mineralized nodule formation, prolonged osteoblast differentiation induction was performed until 21ds with media refreshed twice weekly. The cells were fixed and washed as the ALP staining, then were stained with $1 \%$ alizarin red at $37^{\circ} \mathrm{C}$ for $30 \mathrm{~min}$, washed for observation.

\section{TRAP staining}

Bone marrow macrophages (BMMs) were isolated from the bilateral femur and tibia from normal or CPDtreated $(100 \mathrm{mg} / \mathrm{kg} /$ day) male C57BL/6 mice, seeded per well with $5 \times 10^{4}$ cells in 24 -well tissue treated plates and treated with M-CSF (44 ng/ml) and RANKL (44 ng/ml) for 5 days to generates osteoclasts. Matured osteoclasts were identified with TRAP staining by commercial kit (Sigma). TRAP-positive cells with multi nuclei $(>3)$ are considered as osteoclast. The images were obtained using Leica DMI300B Microscope (Solms, Germany).

For in vivo study, TRAP staining was performed on paraffin sections of tibia. Briefly, the sections were deparaffinized, and dehydrated with $0.02 \mathrm{~g} / \mathrm{ml}$ NaptholEther solution which was diluted with basic stock solution at the ratio of 1:50 for $1 \mathrm{~h}$. The sections were dehydrated and incubated with the diluted NaptholEther solution at $37^{\circ} \mathrm{C}$ for 30 minutes. Then a $0.04 \mathrm{~g} / \mathrm{ml}$ sodium nitrite solution and a $0.05 \mathrm{~g} / \mathrm{ml}$ pararosaniline dye/2 $\mathrm{N}$ hydrochloric acid were mixed before adding to the basic stock solution. The sections were incubated in the mixture for additional 15 minutes. After counter staining and dehydration, the sections were mounted and observed using light microscope (Olympus BX50) and image analysis was performed with software mentioned previously [79]. TRAP activity was quantified by calculating the ratio of the number of TRAP-positive osteoclasts in each representative view area [80].

\section{MTT assay}

Cell viability was analyzed by 3-(4, 5-dimethylthiazol-2-yl)-2,5- Diphenyltetra-zolium bromide (MTT). Briefly, MSCs were seeded at a density of $2 \times 10^{4}$ cells with sextuplicate in 96-well plates. Primary bone MSCs were treated with CPD-Con, CPD-B, CPD-D and CPD-H for 0h,24h,48h,72h, while BMMs were treated CPD working solutions (Control, CPD-Con,CPD-A to CPD-H for $5 \mathrm{ds}$ before adding $20 \mu \mathrm{l} \mathrm{MTT}(5 \mathrm{mg} / \mathrm{ml})$ to each well. The cells were cultured at $37^{\circ} \mathrm{C}$ for $4 \mathrm{~h}$ and the supernatant was discarded. Subsequently, $200 \mu \mathrm{l}$ DMSO was added and the absorbance was read at $570 \mathrm{~nm}$ using a Varioskan TM Flash Multimode Microplate Reader (Thermo Scientific, Waltham, MA, USA). Each assay was independently repeated 3 times and the percentage of cell viability was calculated according to OD ratio. Cell viability $(\%)=\mathrm{OD}$ treated $/ \mathrm{OD}$ control $\times 100 \%$.

\section{Western blot assays}

MSCs were isolated from C57BL/6 mice on day 3 after 7 doses of CPD at $100 \mathrm{mg} / \mathrm{kg}$ injection daily were obtained as plastic adherent cells and subculture to passage 3. MSCs or BMMs isolated from naïve C57BL/6 mice were cultured for adherent, and passage 3 cells in $10 \mathrm{~cm}$ dishes of $75 \%$ confluent were treated with CPD-H for different times. C3H10T1/2 was treated with CPD-H in $10 \mathrm{~cm}$ dishes for different times. The total protein were extracted using the RIPA lysis buffer (Beyotime, Shanghai, China) supplement with $1 \%$ protease inhibitors (Beyotime, Shanghai, China) and 1\% phosphatase inhibitors (SigmaAldrich, St Louis, USA). The cytoplasmic protein, and nuclear protein were extracted from C3H10T1/2 treated with CPD-H for different times using the NE-PER Nuclear and Cytoplasmic Extraction kits according to the manufacturers' instructions.

The protein lysates from bone primary cells or in vivo experiments were subjected to western blot analysis as described previously [7, 76]. The primary antibodies (Cell signaling, MA, USA) against TRAF-3, TRAF-6, NFATc1, RANK, c-Fos, active $\beta$-catenin, Runx2, Cyclin D1, c-Myc, Gsk-3 $\beta$, phospho-GSK-3 $\beta$ and DKK1 were incubated in 5\% non-fat dry milk solution (TBS containing $0.1 \%$ Tween 20). Membranes were washed extensively, and an ECL detection assay was performed using a Super Signal West Dura kit (Beyotime, Shanghai, China).

\section{Real-time RT-PCR}

Lumbar vertebrae of mice after 7 doses of CPD at $100 \mathrm{mg} / \mathrm{kg}$ injection daily were separated and detached cleanly in $2 \mathrm{ml}$ eppendorf tubes containing $1 \mathrm{ml}$ TRIzol and 3 steel balls, grinding in Tissuelyser- 24 according to the manufacturer's instructions. C3H10T1/2 was treated with CPD-H in 6-well plate for different times. Total RNA was prepared using TRIzol reagent (Invitrogen, Mulgrave, Australia) according to the manufacturer's instructions. First-strand cDNA was synthesized from $1 \mu \mathrm{g}$ of total RNA by incubating for $15 \mathrm{~min}$ at $37^{\circ} \mathrm{C}$ with Prime Script RT reagent Kit (Takara) following oligo(dT) priming. After reverse transcription reaction, qRT-PCR was performed by CFX96 PCR system (Bio-Rad) using SYBR1Premix Ex $\operatorname{Taq}^{\mathrm{TM}}$ (Takara, Dalian, China) according to the manufacturer's instructions. Data were analyzed using the comparison $\mathrm{Ct}\left(2^{-\Delta \Delta \mathrm{Ct}}\right)$ method, and expressed as fold change compared with respective control. Each sample was analyzed in triplicate and genes expression of Osteocalcin, ALP, Runx2, Wnt1/4/10b, $\beta$-catenin and Cyclin D1 were normalized to the housekeeping gene $\beta$-actin. The primer sequences for the genes were shown in Table 1.

\section{Semi-quantitative PCR analysis}

Total RNA was isolated from BMMs with TRIzol reagent (Invitrogen, Carlsbad, CA, USA). Superscript 
III transcriptase (Invitrogen) was used to perform the reverse transcription assays according to the standard protocol. The synthesized cDNA in a $20 \mu \mathrm{l}$ volume was applied for regular PCR using Arktik thermal cycler (Thermo Scientific, Vantaa, Finland). The expression of matrix metalloproteinase 9 (MMP9), cathepsin K (Ctsk), tartrate-resistant acid phosphates (TRAP) and carbonic anhydrase II (Car2) and GAPDH genes was measured as previously described [79]. The PCR products were loaded on $2 \%$ agarose gel for electrophoresis analysis. Images of the targeted bands were obtained using GIS-2009 (Tanon Innotech Corporation, Beijing, China).

\section{Statistical analysis}

The GraphPad Prism 5.0 software (GraphPad Software, Inc.) or Excel (2007) was used for the statistical analyses. The results were expressed as mean \pm S.D under each independent condition. Statistical analyses between two groups were assessed by unpaired two-tailed Student's $\mathrm{t}$ test. Statistical analyses among multiple groups were carried out using ANOVA with Bonferroni adjustment. $P$ values less than 0.05 are considered statistically significance.

\section{ACKNOWLEDGMENTS}

The authors wish to acknowledge that research reported in this publication was supported by grants from Shanghai Sailing Program (15YF1412200), the program for the National Natural Science foundation of China (81220108027, 81373667, 81202708, 81774329), Longhua Medical Innovation Team Program (LYCX-01), Innovative Research Team of Ministry of Science and Technology of China (2015RA4002) and Natural Science Foundation of Shanghai (17ZR1430800).

\section{CONFLICTS OF INTEREST}

The authors declare that there are no conflicts of interest.

\section{REFERENCES}

1. Dupuis LL, Roscoe JA, Olver I, Aapro M, Molassiotis A. 2016 updated MASCC/ESMO consensus recommendations: Anticipatory nausea and vomiting in children and adults receiving chemotherapy. Support Care Cancer. 2017; 25:317-321.

2. Goss PE, Muss HB, Ingle JN, Whelan TJ, Wu M. Extended adjuvant endocrine therapy in breast cancer: current status and future directions. Clin Breast Cancer. 2008; 8:411-17.

3. Saarto T, Blomqvist C, Välimäki M, Mäkelä P, Sarna $\mathrm{S}$, Elomaa I. Chemical castration induced by adjuvant cyclophosphamide, methotrexate, and fluorouracil chemotherapy causes rapid bone loss that is reduced by clodronate: a randomized study in premenopausal breast cancer patients. J Clin Oncol. 1997; 15:1341-47.

4. Norton L. Adjuvant breast cancer therapy: current status and future strategies - growth kinetics and the improved drug therapy of breast cancer. Semin Oncol. 1999; 26:1-4.

5. Coleman RE. Current and future status of adjuvant therapy for breast cancer. Cancer. 2003; 97:880-86.

6. Michaud LB, Goodin S. Cancer-treatment-induced bone loss, part 1. Am J Health Syst Pharm. 2006; 63:419-430.

7. Brufsky AM. Cancer treatment-induced bone loss: pathophysiology and clinical perspectives. Oncologist. 2008; 13:187-95.

8. Jonat W, Kaufmann M, Sauerbrei W, Blamey R, Cuzick J, Namer M, Fogelman I, de Haes JC, de Matteis A, Stewart A, Eiermann W, Szakolczai I, Palmer M, et al, and Zoladex Early Breast Cancer Research Association Study. Goserelin versus cyclophosphamide, methotrexate, and fluorouracil as adjuvant therapy in premenopausal patients with node-positive breast cancer: The Zoladex Early Breast Cancer Research Association Study. J Clin Oncol. 2002; 20:4628-35.

9. Shapiro CL, Manola J, Leboff M. Ovarian failure after adjuvant chemotherapy is associated with rapid bone loss in women with early-stage breast cancer. J Clin Oncol. 2001; 19:3306-11.

10. D’Oronzo S, Stucci S, Tucci M, Silvestris F. Cancer treatment-induced bone loss (CTIBL): pathogenesis and clinical implications. Cancer Treat Rev. 2015; 41:798-808.

11. Fogelman I, Blake GM, Blamey R, Palmer M, Sauerbrei W, Schumacher M, Serin D, Stewart A, Wilpshaar W. Bone mineral density in premenopausal women treated for node-positive early breast cancer with 2 years of goserelin or 6 months of cyclophosphamide, methotrexate and 5-fluorouracil (CMF). Osteoporos Int. 2003; 14:1001-1006.

12. Oostra DR, Lustberg MB, Reinbolt RE, Pan X, Wesolowski $\mathrm{R}$, Shapiro CL. Association of osteoprotegerin and bone loss after adjuvant chemotherapy in early-stage breast cancer. Mol Cell Endocrinol. 2015; 402:51-56.

13. Jordan VC, Fritz NF, Tormey DC. Endocrine effects of adjuvant chemotherapy and long-term tamoxifen administration on node-positive patients with breast cancer. Cancer Res. 1987; 47:624-30.

14. Wang TM, Shih C. Study of histomorphometric changes of the mandibular condyles in neonatal and juvenile rats after administration of cyclophosphamide. Acta Anat (Basel). 1986; 127:93-99.

15. Plowchalk DR, Mattison DR. Reproductive toxicity of cyclophosphamide in the C57BL/6N mouse: 1 . Effects on ovarian structure and function. Reprod Toxicol. 1992; 6:411-21. 
16. Plowchalk DR, Mattison DR. Phosphoramide mustard is responsible for the ovarian toxicity of cyclophosphamide. Toxicol Appl Pharmacol. 1991; 107:472-81.

17. Mattison DR, Chang L, Thorgeirsson SS, Shiromizu $\mathrm{K}$. The effects of cyclophosphamide, azathioprine, and 6-mercaptopurine on oocyte and follicle number in C57BL/6N mice. Res Commun Chem Pathol Pharmacol. 1981; 31:155-61.

18. Fan C, Georgiou KR, McKinnon RA, Keefe DM, Howe PR, Xian CJ. Combination chemotherapy with cyclophosphamide, epirubicin and 5-fluorouracil causes trabecular bone loss, bone marrow cell depletion and marrow adiposity in female rats. J Bone Miner Metab. 2016; 34:277-90.

19. Kailajärvi ME, Salminen EK, Paija OM, Virtanent AM, Leino AE, Irjala KA. Serum bone markers in breast cancer patients during 5-fluorouracil, epirubicin and cyclophosphamide (FEC) therapy. Anticancer Res. 2004; 24:1271-74

20. Quach JM, Askmyr M, Jovic T, Baker EK, Walsh NC, Harrison SJ, Neeson P, Ritchie D, Ebeling PR, Purton LE. Myelosuppressive therapies significantly increase proinflammatory cytokines and directly cause bone loss. J Bone Miner Res. 2015; 30:886-897. https://doi.org/10.1002/ jbmr.2415.

21. Georgiou KR, Hui SK, Xian CJ. Regulatory pathways associated with bone loss and bone marrow adiposity caused by aging, chemotherapy, glucocorticoid therapy and radiotherapy. Am J Stem Cells. 2012; 1:205-24.

22. Day TF, Guo X, Garrett-Beal L, Yang Y. Wnt/beta-catenin signaling in mesenchymal progenitors controls osteoblast and chondrocyte differentiation during vertebrate skeletogenesis. Dev Cell. 2005; 8:739-50.

23. Kim JH, Liu X, Wang J, Chen X, Zhang H, Kim SH, Cui J, Li R, Zhang W, Kong Y, Zhang J, Shui W, Lamplot J, et al. Wnt signaling in bone formation and its therapeutic potential for bone diseases. Ther Adv Musculoskelet Dis. 2013; 5:13-31.

24. Bennett CN, Longo KA, Wright WS, Suva LJ, Lane TF, Hankenson KD, MacDougald OA. Regulation of osteoblastogenesis and bone mass by Wnt10b. Proc Natl Acad Sci USA. 2005; 102:3324-29.

25. Macsai CE, Foster BK, Xian CJ. Roles of Wnt signalling in bone growth, remodelling, skeletal disorders and fracture repair. J Cell Physiol. 2008; 215:578-87.

26. Rossini M, Gatti D, Adami S. Involvement of $\mathrm{WNT} / \beta$ catenin signaling in the treatment of osteoporosis. Calcif Tissue Int. 2013; 93:121-32.

27. Albers J, Keller J, Baranowsky A, Beil FT, Catala-Lehnen P, Schulze J, Amling M, Schinke T. Canonical Wnt signaling inhibits osteoclastogenesis independent of osteoprotegerin. J Cell Biol. 2013; 200:537-49.

28. Arioka M, Takahashi-Yanaga F, Sasaki M, Yoshihara T, Morimoto S, Takashima A, Mori Y, Sasaguri T. Acceleration of bone development and regeneration through the Wnt/ $\beta$ catenin signaling pathway in mice heterozygously deficient for GSK-3 $\beta$. Biochem Biophys Res Commun. 2013; 440:677-82.

29. Rao TP, Kühl M. An updated overview on Wnt signaling pathways: a prelude for more. Circ Res. 2010; 106:1798-806.

30. Schoeman MA, Moester MJ, Oostlander AE, Kaijzel EL, Valstar ER, Nelissen RG, Löwik CW, de Rooij KE. Inhibition of GSK3 $\beta$ Stimulates BMP Signaling and Decreases SOST Expression Which Results in Enhanced Osteoblast Differentiation. J Cell Biochem. 2015; 116:2938-46.

31. Gambardella A, Nagaraju CK, O'Shea PJ, Mohanty ST, Kottam L, Pilling J, Sullivan M, Djerbi M, Koopmann W, Croucher PI, Bellantuono I. Glycogen synthase kinase3alpha/beta inhibition promotes in vivo amplification of endogenous mesenchymal progenitors with osteogenic and adipogenic potential and their differentiation to the osteogenic lineage. J Bone Miner Res. 2011; 26:811-821.

32. Nacamuli RP, Fong KD, Warren SM, Fang TD, Song HM, Helms JA, Longaker MT. Markers of osteoblast differentiation in fusing and nonfusing cranial sutures. Plast Reconstr Surg. 2003; 112:1328-35.

33. Lee JM, Libermann TA, Cho JY. The synergistic regulatory effect of Runx2 and MEF transcription factors on osteoblast differentiation markers. J Periodontal Implant Sci. 2010; 40:39-44.

34. Boyle WJ, Simonet WS, Lacey DL. Osteoclast differentiation and activation. Nature. 2003; 423:337-42.

35. Feng X. Regulatory roles and molecular signaling of TNF family members in osteoclasts. Gene. 2005; 350:1-13.

36. Darnay BG, Besse A, Poblenz AT, Lamothe B, Jacoby JJ. TRAFs in RANK signaling. Adv Exp Med Biol. 2007; 597:152-59.

37. Cappellen D, Luong-Nguyen NH, Bongiovanni S, Grenet O, Wanke C, Susa M. Transcriptional program of mouse osteoclast differentiation governed by the macrophage colony-stimulating factor and the ligand for the receptor activator of NFkappa B. J Biol Chem. 2002; 277:21971-82.

38. Takayanagi H, Kim S, Koga T, Nishina H, Isshiki M, Yoshida H, Saiura A, Isobe M, Yokochi T, Inoue J, Wagner EF, Mak TW, Kodama T, Taniguchi T. Induction and activation of the transcription factor NFATc1 (NFAT2) integrate RANKL signaling in terminal differentiation of osteoclasts. Dev Cell. 2002; 3:889-901.

39. Grigoriadis AE, Wang ZQ, Cecchini MG, Hofstetter W, Felix R, Fleisch HA, Wagner EF. c-Fos: a key regulator of osteoclast-macrophage lineage determination and bone remodeling. Science. 1994; 266:443-48.

40. Rho J, Altmann CR, Socci ND, Merkov L, Kim N, So H, Lee O, Takami M, Brivanlou AH, Choi Y. Gene expression profiling of osteoclast differentiation by combined 
suppression subtractive hybridization $(\mathrm{SSH})$ and cDNA microarray analysis. DNA Cell Biol. 2002; 21:541-49.

41. Xiao JH, Zhang Y, Liang GY, Liu RM, Li XG, Zhang LT, Chen DX, Zhong JJ. Synergistic antitumor efficacy of antibacterial helvolic acid from Cordyceps taii and cyclophosphamide in a tumor mouse model. Exp Biol Med (Maywood). 2017; 242:214-22.

42. Han D, Cao C, Su Y, Wang J, Sun J, Chen H, Xu A. Ginkgo biloba exocarp extracts inhibits angiogenesis and its effects on Wnt/ $\beta$-catenin-VEGF signaling pathway in Lewis lung cancer. J Ethnopharmacol. 2016; 192:406-12.

43. Aksnes LH, Bruland OS. Some musculo-skeletal sequelae in cancer survivors. Acta Oncol. 2007; 46:490-96.

44. Bouxsein ML, Boyd SK, Christiansen BA, Guldberg RE, Jepsen KJ, Muller R. Guidelines for assessment of bone microstructure in rodents using micro-computed tomography. J Bone Miner Res. 2010; 25:1468-1486. https://doi.org/10.1002/jbmr.141.

45. Sun S, Guo Z, Xiao X, Liu B, Liu X, Tang PH, Mao N. Isolation of mouse marrow mesenchymal progenitors by a novel and reliable method. Stem Cells. 2003; 21:527-35.

46. Lacey DL, Timms E, Tan HL, Kelley MJ, Dunstan CR, Burgess T, Elliott R, Colombero A, Elliott G, Scully S, Hsu $\mathrm{H}$, Sullivan J, Hawkins N, et al. Osteoprotegerin ligand is a cytokine that regulates osteoclast differentiation and activation. Cell. 1998; 93:165-76.

47. Meier W, du Bois A, Reuss A, Kuhn W, Olbricht S, Gropp M, Richter B, Lück HJ, Kimmig R, Pfisterer J. Topotecan versus treosulfan, an alkylating agent, in patients with epithelial ovarian cancer and relapse within 12 months following 1st-line platinum/paclitaxel chemotherapy. A prospectively randomized phase III trial by the Arbeitsgemeinschaft Gynaekologische Onkologie Ovarian Cancer Study Group (AGO-OVAR). Gynecol Oncol. 2009; 114:199-205.

48. Ackland SP, Anton A, Breitbach GP, Colajori E, Tursi JM, Delfino C, Efremidis A, Ezzat A, Fittipaldo A, Kolaric K, Lopez M, Viaro D, and HEPI 013 study group. Doseintensive epirubicin-based chemotherapy is superior to an intensive intravenous cyclophosphamide, methotrexate, and fluorouracil regimen in metastatic breast cancer: a randomized multinational study. J Clin Oncol. 2001; 19:943-53.

49. Martin M, Villar A, Sole-Calvo A, Gonzalez R, Massuti B, Lizon J, Camps C, Carrato A, Casado A, Candel MT, Albanell J, Aranda J, Munarriz B, et al, Geicam Group S. Doxorubicin in combination with fluorouracil and cyclophosphamide (i.v. FAC regimen, day 1, 21) versus methotrexate in combination with fluorouracil and cyclophosphamide (i.v. CMF regimen, day 1,21) as adjuvant chemotherapy for operable breast cancer: a study by the GEICAM group. Ann Oncol. 2003; 14:833-842.

50. Salas-Ramirez KY, Bagnall C, Frias L, Abdali SA, Ahles TA, Hubbard K. Doxorubicin and cyclophosphamide induce cognitive dysfunction and activate the ERK and AKT signaling pathways. Behav Brain Res. 2015; 292:133-41.

51. Mundy P, da Silva EC, Ledbetter EC. Effects of cyclophosphamide myelosuppression in adult dogs with latent canine herpesvirus-1 infection. Vet Microbiol. 2012; 159:230-35.

52. Sallay K, Sanavi F, Ring I, Pham P, Behling UH, Nowotny A. Alveolar bone destruction in the immunosuppressed rat. J Periodontal Res. 1982; 17:263-74.

53. Cegieła U, Piatek A, Janiec W, Nowińska B, Pytlik M, Folwarczna J, Kwiatkowski Z, Kaczmarczyk-Sedlak I. Effect of cyclophosphamide on bone remodeling in rats. [Article in Polish] Przegl Lek. 2003; 60:329-33.

54. Park SI, Liao J, Berry JE, Li X, Koh AJ, Michalski ME, Eber MR, Soki FN, Sadler D, Sud S, Tisdelle S, Daignault $\mathrm{SD}$, Nemeth JA, et al. Cyclophosphamide creates a receptive microenvironment for prostate cancer skeletal metastasis. Cancer Res. 2012; 72:2522-32.

55. Russell HV, Groshen SG, Ara T, DeClerck YA, Hawkins R, Jackson HA, Daldrup-Link HE, Marachelian A, Skerjanec A, Park JR, Katzenstein H, Matthay KK, Blaney SM, Villablanca JG. A phase I study of zoledronic acid and low-dose cyclophosphamide in recurrent/refractory neuroblastoma: a new approaches to neuroblastoma therapy (NANT) study. Pediatr Blood Cancer. 2011; 57:275-82.

56. Snoeks TJ, Mol IM, Que I, Kaijzel EL, Löwik CW. 2-methoxyestradiol analogue ENMD-1198 reduces breast cancer-induced osteolysis and tumor burden both in vitro and in vivo. Mol Cancer Ther. 2011; 10:874-82.

57. Wu YJ, Muldoon LL, Dickey DT, Lewin SJ, Varallyay CG, Neuwelt EA. Cyclophosphamide enhances human tumor growth in nude rat xenografted tumor models. Neoplasia. 2009; 11:187-95.

58. Yamauchi K, Yang M, Hayashi K, Jiang P, Yamamoto N, Tsuchiya H, Tomita K, Moossa AR, Bouvet M, Hoffman RM. Induction of cancer metastasis by cyclophosphamide pretreatment of host mice: an opposite effect of chemotherapy. Cancer Res. 2008; 68:516-20.

59. Gunjal PM, Schneider G, Ismail AA, Kakar SS, Kucia M, Ratajczak MZ. Evidence for induction of a tumor metastasis-receptive microenvironment for ovarian cancer cells in bone marrow and other organs as an unwanted and underestimated side effect of chemotherapy/radiotherapy. J Ovarian Res. 2015; 8:20.

60. Takada I, Kouzmenko AP, Kato S. Wnt and PPARgamma signaling in osteoblastogenesis and adipogenesis. Nat Rev Rheumatol. 2009; 5:442-47.

61. Ponnapakkam T, Katikaneni R, Nichols T, Tobin G, Sakon J, Matsushita O, Gensure RC. Prevention of chemotherapyinduced osteoporosis by cyclophosphamide with a longacting form of parathyroid hormone. J Endocrinol Invest. 2011; 34:e392-97.

62. Liu J, Wang S, Zhang P, Said-Al-Naief N, Michalek SM, Feng X. Molecular mechanism of the bifunctional role of 
lipopolysaccharide in osteoclastogenesis. J Biol Chem. 2009; 284:12512-23.

63. Burgess TL, Qian Y, Kaufman S, Ring BD, Van G, Capparelli C, Kelley M, Hsu H, Boyle WJ, Dunstan CR, $\mathrm{Hu}$ S, Lacey DL. The ligand for osteoprotegerin (OPGL) directly activates mature osteoclasts. J Cell Biol. 1999; 145:527-38.

64. Lum L, Wong BR, Josien R, Becherer JD, ErdjumentBromage H, Schlöndorff J, Tempst P, Choi Y, Blobel CP. Evidence for a role of a tumor necrosis factoralpha (TNF-alpha)-converting enzyme-like protease in shedding of TRANCE, a TNF family member involved in osteoclastogenesis and dendritic cell survival. J Biol Chem. 1999; 274:13613-18.

65. Mabilleau G, Mieczkowska A, Edmonds ME. Thiazolidinediones induce osteocyte apoptosis and increase sclerostin expression. Diabet Med. 2010; 27:925-32.

66. Sorocéanu MA, Miao D, Bai XY, Su H, Goltzman D, Karaplis AC. Rosiglitazone impacts negatively on bone by promoting osteoblast/osteocyte apoptosis. J Endocrinol. 2004; 183:203-16.

67. Li Q, Zhou X, Shi Y, Li J, Zheng L, Cui L, Zhang J, Wang L, Han Z, Han Y, Fan D. In vivo tracking and comparison of the therapeutic effects of MSCs and HSCs for liver injury. PLoS One. 2013; 8:e62363.

68. Feng X, McDonald JM. Disorders of bone remodeling. Annu Rev Pathol. 2011; 6:121-45.

69. Rehman MU, Tahir M, Ali F, Qamar W, Lateef A, Khan R, Quaiyoom A, Oday-O-Hamiza, Sultana S. Cyclophosphamide-induced nephrotoxicity, genotoxicity, and damage in kidney genomic DNA of Swiss albino mice: the protective effect of Ellagic acid. Mol Cell Biochem. 2012; 365:119-27.

70. Motawi TM, Sadik NA, Refaat A. Cytoprotective effects of DL-alpha-lipoic acid or squalene on cyclophosphamideinduced oxidative injury: an experimental study on rat myocardium, testicles and urinary bladder. Food Chem Toxicol. 2010; 48:2326-2336.
71. Newton HB. Neurological complications of chemotherapy to the central nervous system. Handb Clin Neurol. 2012; 105:903-16.

72. Georgiou KR, King TJ, Scherer MA, Zhou H, Foster BK, Xian CJ. Attenuated Wnt/ $\beta$-catenin signalling mediates methotrexate chemotherapy-induced bone loss and marrow adiposity in rats. Bone. 2012; 50:1223-33.

73. Stretz C, Song X, Killory BD, Ollenschleger MD, Nouh AM. Rheumatoid Meningitis: Diagnostic and Therapeutic Observations. Conn Med. 2016; 80:163-66.

74. Yang Y, Xu S, Xu Q, Liu X, Gao Y, Steinmetz A, Wang N, Wang T, Qiu G. Protective effect of dammarane sapogenins against chemotherapy-induced myelosuppression in mice. Exp Biol Med (Maywood). 2011; 236:729-35.

75. Liu CC, Ho WY, Leu KL, Tsai HM, Yang TH. Effects of S-adenosylhomocysteine and homocysteine on DNA damage and cell cytotoxicity in murine hepatic and microglia cell lines. J Biochem Mol Toxicol. 2009; 23:349-56.

76. Le Pape F, Vargas G, Clezardin P. The role of osteoclasts in breast cancer bone metastasis. J Bone Oncol. 2016; 5:93-95. https://doi.org/10.1016/j.jbo.2016.02.008.

77. Rabello-Gay MN, Carvalho MI, Otto PA, Targa HJ. The effects of age, sex and diet on the clastogenic action of cyclophosphamide in mouse bone marrow. Mutat Res. 1985; 158:181-88.

78. DeLeve LD. Cellular target of cyclophosphamide toxicity in the murine liver: role of glutathione and site of metabolic activation. Hepatology. 1996; 24:830-37.

79. Jules J, Shi Z, Liu J, Xu D, Wang S, Feng X. Receptor activator of NF-\{kappa\}B (RANK) cytoplasmic IVVY535-538 motif plays an essential role in tumor necrosis factor- $\{$ alpha $\}$ (TNF)mediated osteoclastogenesis. J Biol Chem. 2010; 285:37427-35.

80. Zhao D, Shi Z, Warriner AH, Qiao P, Hong H, Wang Y, Feng X. Molecular mechanism of thiazolidinedionemediated inhibitory effects on osteoclastogenesis. PLoS One. 2014; 9:e102706. 